\title{
Population genomics and antimicrobial resistance in Corynebacterium diphtheriae
}

Melanie Hennart ${ }^{1,2}$, Leonardo G. Panunzi ${ }^{1,3}$, Carla Rodrigues ${ }^{1}$, Quentin Gaday ${ }^{4}$, Sarah L. Baines ${ }^{5}$, Marina Barros-Pinkelnig ${ }^{1}$, Annick Carmi-Leroy ${ }^{1,6}$, Melody Dazas ${ }^{1,6}$, AnneMarie Wehenkel ${ }^{4}$, Xavier Didelot ${ }^{7}$, Julie Toubiana ${ }^{1,6,8}$, Edgar Badell $^{1,6}$ and Sylvain Brisse ${ }^{1,6, *}$

${ }^{1}$ Institut Pasteur, Biodiversity and Epidemiology of Bacterial Pathogens, Paris, France. 2 Sorbonne Université, Collège doctoral, F-75005 Paris, France

${ }^{3}$ Institut Français de Bioinformatique, CNRS UMS 3601, Evry, France

${ }^{4}$ Unité de Microbiologie Structurale, Institut Pasteur, CNRS UMR 3528, Université de Paris, F-75015 Paris, France.

${ }^{5}$ Doherty Applied Microbial Genomics, Department of Microbiology \& Immunology, The University of Melbourne at The Peter Doherty Institute for Infection \& Immunity, Melbourne, Victoria, Australia

${ }^{6}$ Institut Pasteur, National Reference Center for Corynebacteria of the diphtheriae complex, Paris, France.

${ }^{7}$ School of Life Sciences and Department of Statistics, University of Warwick, United Kingdom

8 Université de Paris, Department of General Pediatrics and Pediatric infectious diseases, Hôpital Necker-Enfants malades, APHP, Paris France

${ }^{*}$ Corresponding author. Sylvain Brisse. Institut Pasteur, Biodiversity and Epidemiology of Bacterial Pathogens, Paris, France. E-mail: sylvain.brisse@pasteur.fr; Tel: +33 145 688334

Keywords: C. diphtheriae, antibiotic resistance, genome-wide association study, phylogeny, mobile genetic element, biovar

Running title: Genomics of $C$. diphtheriae antimicrobial resistance 


\section{ABSTRACT}

Corynebacterium diphtheriae, the agent of diphtheria, is a genetically diverse bacterial species. Although antimicrobial resistance has emerged against several drugs including first-line penicillin, the genomic determinants and population dynamics of resistance are largely unknown for this neglected human pathogen.

Here we analyzed the associations of antimicrobial susceptibility phenotypes, diphtheria toxin production and genomic features in C. diphtheriae. We used 247 strains collected over several decades in multiple world regions, including the 163 clinical isolates collected prospectively from 2008 to 2017 in France mainland and overseas territories.

Phylogenetic analysis revealed multiple deep-branching sublineages, grouped into a Mitis lineage strongly associated with diphtheria toxin production, and a tox-negative Gravis lineage with few tox ${ }^{+}$exceptions including the 1990s ex-Soviet Union outbreak strain. The distribution of susceptibility phenotypes allowed proposing ecological cutoffs for most of the 19 agents tested, thereby defining acquired antimicrobial resistance. Penicillin resistance was found in $17.2 \%$ of prospective isolates. Four isolates were multidrug resistant (>8 agents), including to penicillin and macrolides. Homologous recombination was frequent $(\mathrm{r} / \mathrm{m}=5)$ and horizontal gene transfer contributed to the emergence of antimicrobial resistance in multiple sublineages. Genome-wide association mapping uncovered genetic factors of resistance, including an accessory penicillinbinding protein (PBP2m) located in diverse genomic contexts. Gene pbp2m is widespread

51 in other Corynebacterium species and its expression in C. glutamicum demonstrated its

52 effect against several beta-lactams. A novel 73-kb $C$. diphtheriae multi-resistance 53 plasmid was discovered.

54 This work uncovers the dynamics of antimicrobial resistance in C. diphtheriae in the 55 context of phylogenetic structure, biovar and diphtheria toxin production, and provides a blueprint to analyze re-emerging diphtheria. 


\section{INTRODUCTION}

58

59

60

61

62

63

64

65

66

67

68

69

70

71

72

73

74

75

76

77

78

79

80

81

82

83

84

85

86

87

Diphtheria, if untreated, is one of the most severe bacterial infections of humans. It typically affects the upper respiratory tract causing pseudomembrane formation, sometimes leading to suffocation and death. The infection can be complicated by toxinic symptoms, caused by the diphtheria toxin. Other forms of disease are skin and invasive infections, including endocarditis ${ }^{1,2}$.

The agent of diphtheria is Corynebacterium diphtheriae, a member of the phylum Actinomycetes ${ }^{34}$. The diphtheria toxin, encoded by the tox gene, is carried by lysogenized corynephages within the chromosome of some $C$. diphtheriae strains ${ }^{5,6}$. Concern exists about the possibility of lysogenic conversion of previously non-toxigenic strains during colonization, infection or transmission chains 7. However, knowledge on the microevolutionary dynamics between tox-positive and tox-negative strains is limited. The high genetic diversity of $C$. diphtheriae strains underlies their variable colonization, adhesion and pathogenicity properties ${ }^{8-10}$. Although three main biovars (Mitis, Gravis and Belfanti) are distinguished since the 1950s, their phylogenetic relationships are poorly defined ${ }^{11-13}$.

Diphtheria used to be one of the deadliest infections in young children, but has been largely controlled by vaccination with the highly effective toxoid vaccine ${ }^{14}$. Even so, thousands of cases of diphtheria are still reported annually ${ }^{15}$, and large outbreaks can quickly follow the disruption of public health systems 14,16-18. In countries with high vaccination coverage, diphtheria cases are associated with travel and migration from endemic regions ${ }^{19-21}$. As diphtheria vaccination is performed using an inactivated form of diphtheria toxin, it is not considered to prevent asymptomatic colonization and silent transmission of the pathogen, which still circulates and is the object of intense epidemiological surveillance ${ }^{4}$. However, vaccine preparations may include other antigens and the impact of vaccination on $C$. diphtheriae evolution deserves further studies ${ }^{22}$.

Clinical management of infections with toxigenic isolates includes treatment with diphtheria antitoxin (DAT), which can prevent or reduce toxinic complications ${ }^{4}$. Nevertheless, antimicrobial treatment is critical in clinical management of both toxpositive and tox-negative infections, as it contributes to the elimination of the bacteria 
within the patient and limits transmission to novel individuals ${ }^{23}$. With DAT production being threatened ${ }^{24}$, antimicrobial treatment might become even more critical in diphtheria therapy.

Penicillin is the first-line therapeutics to treat diphtheria, with erythromycin being recommended in case of allergy ${ }^{25}$. Both antimicrobial agents are effective for the treatment of diphtheria 23,26 . However, reduced susceptibility or full resistance of C. diphtheriae to penicillin has been reported from multiple world regions ${ }^{27-31}$. Resistance against other antimicrobial agents including erythromycin has also been reported 23,26,27,32-35. Although rare, multidrug resistant $C$. diphtheriae have been described $26,27,32,35,36$.

Antimicrobial resistance genes have been described in $C$. diphtheriae, including the erythromycin resistance gene ermX on plasmid pNG2 37 and genes dfrA16, qacH and sul1 carried on a class 1 integron, mobilized by IS6100 ${ }^{38}$. However, the prevalence and phylogenetic distribution of resistance genes in $C$. diphtheriae clinical isolates are unknown. Six chromosomal penicillin-binding proteins (PBP) have been reported in C. diphtheriae ${ }^{39}$, but so far no association between pbp or other genetic variation and penicillin resistance has been described. Understanding the genetic basis of antimicrobial resistance in $C$. diphtheriae would improve our ability to diagnose and track its spread.

The aims of this study were (i) to characterize antimicrobial resistance phenotypes in a large collection of $C$. diphtheriae strains with diverse geographical and temporal origins, and to uncover genomic determinants of resistance; and (ii) to analyze the population structure of $C$. diphtheriae and define associations between antimicrobial resistance, diphtheria toxin production, biovars and phylogenetic sublineages. 


\section{1}

112

113

114

115

116

117

118

119

120

121

122

123

124

125

126

127

128

129

130

131

132

133

134

135

136

137

138

139

140

141

\section{RESULTS}

\section{Provenance and microbiological characteristics of $\boldsymbol{C}$. diphtheriae isolates}

We studied 247 C. diphtheriae strains of diverse geographic and temporal origins (Figure 1). This collection included 163 isolates prospectively collected between 2008 and 2017 from French mainland and overseas territories, 15 older (1981-2006) French clinical isolates, 65 ribotype reference strains ${ }^{40}$ and 4 other reference strains. All isolates were confirmed as $C$. diphtheriae (excluding $C$. belfantii and $C$. rouxii) based on an average nucleotide identity (ANI) value higher than $96 \%$ with the C. diphtheriae type strain NCTC11397'.

Approximately one third ( $n=78,32 \%$ ) of isolates were tox-positive (as defined by the detection of the tox gene by PCR), whereas the remaining 169 isolates (68\%) were toxnegative. The proportions of tox-positive isolates were $42 \%, 34 \%$ and $2 \%$ among reference strains, 2008-2017 clinical isolates and older clinical isolates, respectively (Figure S1). Of the 78 tox-positive isolates, 17 (21.8\%) were negative for toxin production and thus correspond to non-toxigenic toxin-gene bearing (NTTB) isolates. Six of the NTTB isolates had a stop codon within the tox gene sequence (Table S1; Table S2). However, for the 11 remaining strains, we found no explanation for the observed lack of toxin production.

Upon biotyping, $154(62.3 \%)$ isolates belonged to biovar Mitis, 87 to biovar Gravis $(35.2 \%)$ and $6(2.4 \%)$ to biovar Belfanti (Table S1). Biovar proportions were similar among the three datasets. Mitis isolates were more frequently tox-positive than Gravis isolates (56/154 versus 18/87, chi-squared test, $p$-value 0.01; Figure S1). Among toxpositive isolates, NTTB were more frequent among Mitis isolates $(13 / 56,23.2 \%)$ than among Gravis isolates (1/18,5.6\%) although this difference was not statistically significant ( $p$-value 0.09). Three out of four tox-positive Belfanti isolates were NTTB.

\section{Phylogenetic structure of $C$. diphtheriae and distribution of the toxin gene}

To infer a phylogenetic tree, we first aimed to detect and remove homologous recombination events among $C$. diphtheriae genomic sequences. ClonalFrameML inferred a relative rate of recombination to mutation (R/theta) of 0.86 , with an average 
142 length of recombination segments (delta) of $287 \mathrm{bp}$. The mean genetic distance between

143 donor and recipient of recombination (nu) was 0.02 substitutions per nucleotide position,

144 resulting in a relative impact of recombination to mutation ( $\mathrm{r} / \mathrm{m}=\mathrm{R} /$ theta $\times$ delta $\times \mathrm{nu}$ ) of 5.01 .

145 The recombination-corrected phylogeny (Figure 2; Figure S2) was star-like, with a

146 multitude of sublineages branching off deeply. The deepest branching sublineages

147 corresponded to two ribotype reference strains of biovar Mitis: CIP107521 (ribotype

148 Dagestan) and CIP107534 (ribotype Kaliningrad). Remarkably, isolates of biovars Mitis

149 and Gravis were mostly distributed in two distinct branches of the tree. We therefore

150 named the two major branches, lineage Mitis (156 strains, of which 86\% were of biovar

151 Mitis) and lineage Gravis (91 strains, of which 77\% were of biovar Gravis). The Gravis

152 lineage branched off from within the Mitis lineage (Figure 2). Reference strains PW8 and

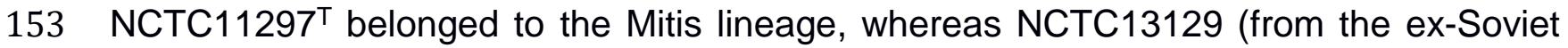

154 Union 1990's outbreak) and NCTC10648 belonged to the Gravis lineage. The Belfanti

155 isolates were scattered in three distinct sublineages within the Mitis lineage and one 156 within the Gravis lineage.

157 The isolates carrying the tox gene belonged mostly to the Mitis lineage (68 of 78,

158 87.2\%), in which they were distributed in multiple sublineages. In the Mitis lineage, 69

159 (44.2\%) were tox-positive. In contrast, within the Gravis lineage, only 10 (11\%) isolates

160 were tox-positive, and they corresponded to the earliest-branching Gravis sublineages

161 with only one exception. Interestingly, this exception corresponded to the large ex-Soviet

162 Union outbreak in the 1990s (Figure 2). This phylogenetic pattern is consistent with an

163 evolutionary scenario where Mitis is the ancestral biovar of $C$. diphtheriae and where

164 Gravis evolved from the Mitis lineage as an initially tox-positive sublineage, with

165 subsequent loss of the toxin gene. In this scenario, the ex-Soviet Union outbreak

166 sublineage would have re-acquired the tox gene. All NTTB isolates belonged to the Mitis

167 lineage except strain CIPA99 (ribotype Rhone, biovar Belfanti; Figure 2), and they were

168 distributed in multiple sublineages, showing convergent evolution towards the loss of toxin

169 production.

170

171 Genetic events linked to biovar status 
Biovar Mitis and Gravis are distinguished by the ability to utilize glycogen (positive in

173 Gravis, negative in Mitis). The spuA gene, which codes for a putative alpha-1,6-

174 glycosidase, was reported as being specific for biovar Gravis isolates ${ }^{41}$. Our genome-

175 wide association study (GWAS) of accessory genes with the biovar phenotype revealed

176 a strong association of a cluster of genes that includes spuA (DIP357; Figure S3) with

177 biovar Gravis isolates. This association was stronger within the Gravis lineage; in contrast

178 within the Mitis lineage, few of the biovar Gravis isolates possessed spuA (Figure 2).

179 GWAS analysis of core SNPs further demonstrated that a SNP (at position 324,487 ,

180 Figure S3) downstream of the spuA cluster insertion point was also associated with

181 biovar, suggesting homologous recombination among core genes as a mechanism for

182 the spuA cluster insertion event.

183 The nitrate reductase activity differentiates Mitis and Gravis isolates, which are 184 positive, from Belfanti isolates, which are nitrate-negative. We found that the nitrate 185 reduction narKGHJI gene cluster ${ }^{41}$ was disrupted in three of the six isolates assigned to 186 the biovar Belfanti: strains FRC0480 and FRC0481 had a G to A mutation at position 675 187 of the narG gene, leading to a stop codon; whereas in strain CIPA99, approximately 100 188 nucleotides were inserted at position 446 in narG. No molecular explanation was found 189 for the lack of nitrate reductase ability of the three other Belfanti strains when scrutinizing 190 the narKGHJI gene cluster and adjacent molybdenum cofactor biosynthesis genes ${ }^{42}$.

\section{Antimicrobial susceptibility variation}

193 Susceptibility to 19 antimicrobial agents was determined for the 247 clinical isolates 194 and reference strains (Table S3). For each agent, the distribution of zone diameter (ZD) 195 values (Figure 3) revealed a predominant mode located towards the right end of the 196 distribution. This mode likely corresponds to the natural susceptibility distribution within 197 the $C$. diphtheriae population and was used to define tentative epidemiological cutoffs 198 (ECOFF, also called ecological cutoff ${ }^{43}$ ). The proposed ECOFFs and their comparison 199 with clinical breakpoints are presented in Table S4. For each antimicrobial agent except 200 cefotaxime, this approach led to the identification of outsider strains with potentially 201 acquired resistance (Figure 3 ). 
Penicillin was exceptional in that the predominant susceptible mode (centered around $36 \mathrm{~mm}$ ) was less neatly defined, due to partial overlap with a second mode of smaller diameter values centered around $24 \mathrm{~mm}$. This second mode corresponds mostly to the 'intermediate' interpretative category $(18<=Z D<29 \mathrm{~mm})$ but also overlaps with the 'resistant' category $(<18 \mathrm{~mm})$. The distribution of ZD values for tetracycline also showed a clear second mode. For multiple other agents (amoxicillin, oxacillin, imipenem, kanamycin, rifampicin, ciprofloxacin, clindamycin and more evidently sulfonamide, trimethoprim and the trimethoprim-sulfamethoxazole combination), outsider strains had 210 the minimal diameter (6 $\mathrm{mm}$, corresponding to growth at the disk contact). For 211 trimethoprim, we observed both a mode centered around $14 \mathrm{~mm}$ and a group of even 212 more resistant outliers with growth at disk contact.

213 Antimicrobial resistance levels were similarly distributed between tox-positive and tox214 negative isolates (Figure S4) as well as between the two main phylogenetic lineages or 215 biovars (Figure S5; Table S1).

Resistance rates were $17.2 \%, 2.5 \%$ and $2.5 \%$ for penicillin, amoxicillin and erythromycin, respectively, among the prospectively collected 2008-2017 clinical isolates (Figure 4). Reference strains were generally susceptible to most agents, including penicillin, but were partially resistant to tetracycline (18\%) and sulfonamide (35\%). The resistance profiles distribution showed that approximately half $(121 / 247)$ of the strains

222 had a fully susceptible phenotype, whereas four isolates were multidrug resistant ( $>8$ 223 agents; Figure 4 inset). Notably, these four isolates were resistant at the same time to 224 penicillin and macrolides, and two of them (FRC0402 and FRC0466) additionally had a 225 reduced susceptibility to amoxicillin. Two multidrug resistant isolates were collected from 226 a foot arch wound and in respiratory carriage in the same patient (French mainland, with 227 recent travel from New Caledonia). The two others came from a patient living in La 228 Réunion Island (FRC0402) and from a patient living in Paris, who had recently traveled 229 to Tunisia (FRC0466). Isolates from La Réunion Island and mainland France showed 230 resistance to multiple antimicrobial agents more often than isolates from other geographic 231 origins (Figure 1A inset). 


\section{Genomic associations with antimicrobial resistance phenotypes}

234 We first searched for the presence in the genomic sequences, of previously described antimicrobial resistance genes (ARGs). This approach led to detection of 12 ARGs (Table

236 S5). We identified three tetracycline resistance genes (tetW, tet33 and tetO), four 237 aminoglycoside resistance genes [aph(3')-la, aph(3')-lb, aph(6)-Id, and aadA1 = ant(3')238 la], and also ermX, dfrA16, dfrA15b, dfrA1 and sul1 genes. We observed a strong 239 correlation between the presence of ARGs and the expected resistance phenotypes 240 (Figure S6), particularly for ermX (macrolide resistance), sul1 (sulfonamide resistance) 241 and $a p h\left(3^{\prime}\right)-l a$ (kanamycin resistance). In strains FRC0137 and FRC0375, this latter gene 242 was linked to aph(3')-Ib (strA), aph(6)-Id (strB) and ermX on a Tn5432-like genomic region 243 with an IS1249 insertion sequence ${ }^{44}$. The phylogenetic distribution of ARGs (Figure 5) 244 revealed their presence in multiple unrelated sublineages, consistent with independent 245 acquisitions by horizontal gene transfer. Gene ermX was present either in proximity to 246 gene pbp2m (see below) or in a fragmented insertion-sequence rich accessory region. 247 Gene dfrA16 was associated with sul1 on a reported ${ }^{38}$ class 1 integron (see below, 248 resistance plasmid section). Tetracycline resistance was associated either with the 249 ribosomal protection protein genes tet(O) or tet $(\mathrm{W})$, or with the efflux pump gene tet33. 250 These three genes were present in distinct strain subsets and appear to contribute 251 independently to tetracycline resistance in C. diphtheriae (Figure 5); they were mostly 252 associated with insertion sequences but not with other ARGS.

253 Next, in order to identify novel genetic determinants potentially associated with 254 antimicrobial resistance in C. diphtheriae, a GWAS approach was followed, based on 255 either core genome SNPs or accessory gene presence/absence. SNPs that were strongly 256 associated with ciprofloxacin, trimethoprim and rifampicin resistance were identified 257 within genes for gyrase subunit A, dihydrofolate reductase and RNA polymerase subunit 258 B, respectively (Table S5; Figure S7), consistent with known mechanisms and validating 259 our approach. SNPs were also found to be associated with penicillin, kanamycin and 260 tetracycline resistance (Table S5), but in these cases the functional attribution is 261 undefined. No association was found for penicillin-resistance within the core PBP genes 262 using the genome-wide approach. However, using a concatenation of the amino-acid 263 sequences of the seven identified putative PBPs of $C$. diphtheriae, we identified amino- 
264 acid positions that were statistically associated with penicillin resistance (Figure S8). The 265 identified positions were mapped onto the predicted functional domains of the different 266 PBPs (Figure S9) revealing several mutation hotspots. A number of significant SNPs were found within the transpeptidase (TP) domains of the different PBPs, but none of the mutations mapped to the conserved transpeptidation motif. Other mutations were found outside the TP domains, for instance in the transglycosylase and PASTA domains of PBP1b or in the dimerization domain of PBP2b.

GWAS analysis of accessory genes demonstrated significant associations with 272 phenotypic resistance. Associated genes included those mentioned above for 273 erythromycin, tetracycline, kanamycin, sulfonamide and trimethoprim (Table S5). In

274 addition, an accessory penicillin-binding protein gene (which we name pbp2m) was 275 strongly associated with penicillin resistance. This gene is described in more detail below.

Discovery of a novel penicillin-binding protein (PBP2m) associated with penicillin resistance

The novel PBP gene pbp2m was observed in 11 isolates, 8 of which were penicillin resistant with minimum inhibitory concentrations (MIC) ranging from 0.19 to $1.5 \mathrm{mg} / \mathrm{L}$. Two of these isolates were also resistant to amoxicillin and one was in addition resistant to oxacillin (Table S4). The phylogenetic distribution of $p b p 2 m$-positive strains was compatible with multiple independent acquisitions of the gene through horizontal gene transfer (Figure 5). Sequence analysis showed that the newly identified PBP2m is almost identical to PBP2c from $C$. jeikeium, a class B PBP with an N-terminal signal peptide followed by a lipobox domain and the C-terminal transpeptidase domain (Figure S9). The C. jeikeium PBP2c is a low affinity PBP and was associated with beta-lactam resistance in C. jeikeium ${ }^{45}$.

To demonstrate the role of PBP2m in penicillin resistance, its gene was PCR amplified from FRC0402 and cloned into the pTGR5 plasmid (Figure S10).

291 Transformation of the plasmid into C. glutamicum strain ATCC 13032 raised the MIC for 292 penicillin from 0.125 to $1.5 \mathrm{mg} / \mathrm{L}$, and the MICs of the other beta-lactams amoxicillin, 293 cefotaxime and oxacillin also increased importantly (Figure 6; Table S6). In contrast, 294 MICs of non-beta-lactam agents were not changed. Imipenem was less effective against 
295 the transformant based on disk diffusion but not based on E-test. Transformation with the

296 empty plasmid used as control did not affect the MIC of any agent. These results show

297 that PBP2m confers resistance to a broad range of beta-lactams.

Discovery of a multidrug resistant conjugative plasmid carrying the gene pbp2m

Strain FRC0402, a tox-negative isolate from La Réunion Island, stood out as being resistant to 12 agents (Figure 4 inset). In addition to pbp2m, this isolate carried genes sul1, ermX and dfrA16 and a tetA family tet(Z)-like (71\%) tetracycline efflux gene. To define the genomic context of resistance genes, a complete genome sequence was obtained. The assembly revealed a chromosome of 2,397,465 bp and a circular plasmid of 73,763 bp (Figure 7), which we propose to name pLRPD (for large resistance plasmid of $C$. diphtheriae).

The pbp2m gene was located on the large plasmid in a region comprising three other genes, a blaB beta-lactamase family gene, a LysR family regulator gene and the ermX gene, flanked by two insertion sequences (IS1628) of the IS6 family (Figure 7). With disparate direct repeat sequences, it remains unclear if this region represents a single

311 transposable unit or a mosaic of gene acquisition events in pLRPD. A nearly identical PBP was observed in $78 \%$ of publicly available $C$. jekeium genomes, in $57 \%$ of $C$. striatum genomes and in multiple other Corynebacterium genomes (Table S7). However, the genetic context of PBP2m was highly variable in $C$. diphtheriae and among other Corynebacterium species (Figure S11). A putative transposable PBP-containing unit (PCU) comprising genes pbp2m, blaB and lysR, commonly flanked by IS3503 (IS256 family) with a fragment identified in pLRPD (Figure 7), appeared to be highly conserved and was associated variably with ermX in $C$. diphtheriae and with a helicase in C. diphtheriae and other Corynebacterium species. The PCU was sometimes found in 2 or 3 tandem copies and was chromosomally located in most genomes.

321 Further elements carried by pLRPD included an integron carrying genes dfrA16, 322 qacL, sul1, as well as elements of a putative conjugation apparatus gene cluster (Figure 323 7). Our conjugation experiments aiming to demonstrate the transfer of pLRPD into 324 recipient $C$. diphtheriae isolates failed. This plasmid was not found in other $C$. diphtheriae 325 strains. 
Strains of $C$. diphtheriae that are resistant to antimicrobial therapy may compromise the management of diphtheria cases and the control of pathogen transmission. Here we aimed to define the genomic determinants of resistance to penicillin and other antimicrobial agents in $C$. diphtheriae, and to analyze the relationships of resistance with diphtheria toxin production, biochemical variants and phylogenetic sublineages. To this aim, we characterized phenotypically and genotypically, a large sample of $C$. diphtheriae isolates from diverse geographic and temporal origins. We confirmed that the species is made of multiple phylogenetic sublineages $9,13,46$ and showed that homologous recombination contributes five times more to their diversification than mutation, consistent with previous evidence of recombination in $C$. diphtheriae populations ${ }^{12,13}$.

Historically, $C$. diphtheriae isolates have been classified into three main biovars, but the links between biovars and phylogenetic structure have remained obscure. Whereas previous work concluded on the absence of an association ${ }^{11}$, our phylogenetic analyses reveal that Gravis and Mitis, the two main biovars of $C$. diphtheriae, are associated 342 strongly with two phylogenetic lineages. Lineage Gravis appears to have acquired ancestrally a gene cluster comprising the extracellular glycogen debranching enzyme gene spuA ${ }^{41}$. Although the Gravis phenotype is largely associated with spuA within 345 lineage Gravis, other genomic determinants of glycogen utilization remain to be discovered within the Mitis lineage. Whereas most of our biovar Belfanti isolates were excluded from this work because they belonged to $C$. belfantii or $C$. rouxii, a few Belfanti isolates did belong to $C$. diphtheriae. Our results show that biotyping is subject to parallel evolution and has limited epidemiological typing value.

The most important factor of $C$. diphtheriae pathogenicity is the diphtheria toxin. Despite early realization that it is encoded on a prophage ${ }^{5}$, few studies have investigated 352 the phylogenetic distribution of the tox gene in $C$. diphtheriae ${ }^{8,9}$. Here, we show that tox353 positive strains mainly belong to the Mitis lineage and to early-diverging branches of the 354 Gravis lineage. The distribution of tox-positive isolates into multiple Mitis sublineages is 355 strongly indicative of independent acquisitions of the toxin gene. Alternately, this pattern 356 might result from initial acquisition of the tox gene, followed by secondary loss in multiple 
sublineages. The phylogenetic pattern is also consistent with an ancestral presence of

358 the tox-bearing phage in the Gravis lineage, with subsequent loss of the tox gene in the 359 branch leading to the ancestor of most Gravis isolates. Future work should investigate 360 the dynamics of the lysogenic corynephages and molecular determinants of their 361 sublineage distribution. One important open question is the likelihood of tox-negative 362 strains acquiring the tox gene during colonization, infection or short-term epidemiological 363 timeframes ${ }^{7}$.

364 Remarkably, except for early-diverging sublineages, only one Gravis sublineage was 365 found to carry the tox gene. This sublineage happens to correspond to the largest outbreak in recent times, which occurred in Newly Independent States of the ex-Soviet Union in the 1990s ${ }^{13,14,39}$. This sublineage, which comprises the ST8 reference strain NCTC13129, is genetically distant from other tox-positive lineages, which belong to the Mitis lineage. Hence, its antigenic structures or other pathogenicity properties may have diverged from those of more common tox-positive isolates, which might have contributed

371 to its exceptional transmission in the 1990s, in addition to the decline in vaccine coverage 14. Of note, biovar Gravis was named to reflect a perceived higher severity of infection compared to diphtheria cases caused by biovar Mitis isolates ${ }^{47,48}$. Recently it was shown that most diphtheria vaccines contain, besides the anatoxin, multiple other $C$. diphtheriae immunogens ${ }^{22}$. The impact of vaccination on the evolution of $C$. diphtheriae populations, and possible variations of cross-protection as a function of strain diversity, are currently undefined. This work provides a framework onto which future studies can build to address this important question.

Although antimicrobial resistant $C$. diphtheriae strains have been reported on numerous occasions ${ }^{23,32}$, knowledge on antimicrobial resistance in $C$. diphtheriae is largely fragmented and suffers from lack of harmonization. Breakpoints used to define resistance vary according to world region and have changed over time within single countries ${ }^{49}$. The lack of consensus on the definition of resistance restricts our ability to define the magnitude of the problem and its global significance.

We aimed to define biologically meaningful cutoffs ${ }^{43}$ based on susceptibility 387 phenotypes distributions, taking advantage of our large and diverse sample. Our data 
388 allowed us to propose tentative ecological cutoffs for C. diphtheriae. Clearly, this 389 approach should in the future be extended to MIC values and should use larger and more 390 diverse strain collections. Nevertheless, our analyses suggest that non-susceptibility to 391 at least one antimicrobial agent was acquired by half of $C$. diphtheriae strains, regardless 392 of lineage, biovar or toxigenic status. This study further suggests that acquired resistance 393 to penicillin, the first line therapy against diphtheria, is far from being rare, affecting >15\% 394 of $C$. diphtheriae isolates collected in the last decade in France and its overseas 395 territories. The high prevalence of resistance to penicillin, tetracycline and 396 trimethoprim/sulfamethoxazole found here are consistent with susceptibility surveys of 397 recent $C$. diphtheriae isolates in Algeria ${ }^{29}$, Indonesia ${ }^{31,50}$ and India ${ }^{51}$. Many high-income 398 countries such as France have chosen to use amoxicillin as the first choice for antibiotic 399 therapy ${ }^{52}$, as this molecule remains highly active. Still, widespread penicillin resistance 400 is concerning, since diphtheria mainly occurs in resource-poor settings where penicillin G 401 is largely used. In contrast, resistance to erythromycin and other macrolides remains rare. 402 Our results call for concerted research into the magnitude of the antimicrobial resistance 403 threat in C. diphtheriae.

404

Knowledge of the genetic mechanisms of antimicrobial resistance is critical for 406 defining appropriate treatments, refining diagnostics and conducting epidemiological studies of antimicrobial resistance. Resistance genes to several antimicrobial classes have been described in $C$. diphtheriae ${ }^{37,38,53}$, while additional genes described in other Corynebacterium species ${ }^{54}$ might also be present in $C$. diphtheriae. Here, we defined the prevalence and phylogenetic distribution of previously reported and newly identified resistance determinants in $C$. diphtheriae. We demonstrate the co-occurrence of 412 resistance phenotypes and genes, suggesting a causative link in multiple instances. We 413 further show that resistance genes have been acquired independently in multiple 414 sublineages, demonstrating a dynamic resistome in $C$. diphtheriae. In addition, we 415 demonstrate an association between alterations in chromosomally encoded targets and 416 phenotypic resistance for quinolone, trimethoprim and rifampicin. Fluoroquinolone 417 resistance was previously linked to mutations in the gyrA gene in C. amycolatum ${ }^{55}$, 418 C. striatum ${ }^{56}$ and $C$. belfantii 57 but seemingly never for $C$. diphtheriae. Finally, we 
demonstrate the co-occurrence within some strains, of multiple resistance determinants and uncover a previously undescribed large resistance plasmid in $C$. diphtheriae. The mechanism of genetic transfer of this plasmid remain to be investigated. This work provides a first overview of the $C$. diphtheriae resistome and will facilitate further studies into the evolutionary emergence of multiresistant $C$. diphtheriae strains.

Mechanisms of penicillin resistance in $C$. diphtheriae have never been described, to our knowledge. Here we discovered an accessory PBP (PBP2m), which was experimentally shown to confer resistance to penicillin and other beta-lactam antimicrobial agents. Its distribution in multiple sublineages, and its presence in other Corynebacterium species, clearly demonstrates its horizontal transfer, and we revealed a multiplicity of genomic contexts in which it is found within Corynebacterium. PBP2m is a putative low affinity PBP, which would explain why it is less affected by beta-lactam antibiotics. Further studies on the expression, antimicrobial resistance spectrum and mechanism of action of PBP2m are warranted.

The seven chromosomal PBPs of $C$. diphtheriae (including the newly annotated PBP4b) were investigated to identify amino acid sequence polymorphisms associated with penicillin resistance. Although several alterations were significantly associated, none were directly implicated with the catalytic residues of the transpeptidase or transglycosylase domains. The association with resistance may not be directly linked to these catalytic residues but could be due to secondary sites that are thought to interfere with beta-lactam ligand binding. While biochemical and structural studies are necessary to understand how these mutations affect penicillin susceptibility, we postulate that some changes in these domains could lead to allosteric effects ultimately resulting in betalactam resistance, as described for Staphylococcus aureus PBP2a ${ }^{58,59}$ or Streptococcus pneumoniae PBP2x ${ }^{60}$. Other SNPs might simply have been hitchhiking due to their physical linkage with functionally important SNPs ${ }^{61}$.

\section{Conclusion}

As a result of vaccination and antitoxin therapy, diphtheria has fallen from a main killer of young children to a largely controlled disease. However, in recent years vaccination rates have dropped in several settings afflicted by conflicts or economic crises, and the 
450 lack of availability of diphtheria antitoxin is becoming critical. Antimicrobial therapy is an

451 increasingly important component of diphtheria control, but its efficacy is jeopardized by

452 emerging resistance. Here we contributed to define the magnitude of this issue and

453 provide novel insights into its genomic underpinnings. We also provide fresh views on the

454 population structure of the $C$. diphtheriae species, and associations between traditional

455 biochemical characterization of strains into biovars, the distribution of toxigenic isolates,

456 and the population dynamics of antimicrobial resistance within C. diphtheriae. 
METHODS

458

\section{Corynebacterium diphtheriae isolates and strains}

A collection of 247 C. diphtheriae isolates were included (Table S1), corresponding to three subsets. First, we included 163 clinical isolates (Recent clinical isolates subset, 462 Table S1) collected prospectively between 2008 and 2017 by the French National 463 Reference Center for Corynebacteria of the Corynebacterium diphtheriae complex (NRC464 CCD). These isolates represented all isolates received at the NRC-CCD that corresponded to the

C. diphtheriae species

(C. belfantii,

C. ulcerans, $C$. pseudotuberculosis or other corynebacteria were excluded). They were collected from cutaneous $(n=136)$, respiratory $(n=23)$ and other infections (bones, blood; $n=4)$. Of these, 74 were from Mainland France and 89 from French overseas territories, including Mayotte $(n=50)$, New Caledonia $(n=19)$, La Réunion island $(n=11)$, French Guiana $(n=4)$, French Polynesia $(n=3)$ and Guadeloupe $(n=1)$; one isolate received from Institut Pasteur in Madagascar was also included (Figure 1). Four isolates from New Caledonia collected between 2002 and 2006 (02-0322, 02-0338, 03-1641 and 06-1569) were included in a previous study ${ }^{62}$; the trimethoprim and sulfamethoxazole-resistant isolate FRC0024 was previously shown to harbor an integron with gene $\operatorname{drf} A 16^{38}$.

Second, we included 15 clinical isolates collected in France between 1981 and 1991, 11 of which had been deposited in the Collection de I'Institut Pasteur (CIP; Historical clinical isolates subset in Table S1).

Third, to increase the genetic diversity and geographic range of the sample, the 65 available reference strains of ribotypes that belong to $C$. diphtheriae were included ${ }^{40}$. These reference strains represent an international collection of isolates collected over several decades and originating from multiple world regions including the Americas, 482 Europe, Asia, Africa, and Oceania. Our subcultures of these strains were controlled for tox gene presence, toxin production and biovar, leading to modifications of published characteristics in some instances (Table S1; Figure 1). Finally, four reference strains were included: strain NCTC13129, which is used as genomic sequence reference ${ }^{39}$; strain NCTC10648, which is used as the tox-positive and toxinogenic reference strain in 487 PCR and Elek tests, respectively; strain NCTC11397' ${ }^{\top}$, which is the taxonomic type strain 
488 of the $C$. diphtheriae species; and the vaccine production strain PW8, which corresponds 489 to CIP A102 ${ }^{63}$. This third subset is referred to as "Ribotype and reference strains" subset 490 (Table S1).

\section{Bacterial cultures, identification and biovar}

493 Bacteria were cultivated on Trypto-Casein-Soy (TCS) agar during $24 \mathrm{~h}$ at $35-37^{\circ} \mathrm{C}$. 494 Bacterial identification was performed at the NRC-CCD as described previously ${ }^{64}$ by 495 multiplex polymerase chain reaction (PCR) combining a $d t x R$ gene fragment specific for 496 C. diphtheriae and a multiplex PCR that targets a fragment of the pld gene specific for 497 C. pseudotuberculosis, the gene rpoB (amplified in all species of the $C$. diphtheriae 498 complex) and a fragment of 16S rRNA gene specific for $C$. pseudotuberculosis and $C$. 499 ulcerans. Isolates collected since 2014 were confirmed as $C$. diphtheriae by matrix500 assisted laser desorption-ionization time-of-flight mass spectrometry (MALDI-TOF MS) 501 using Bruker technology. In order to exclude strains initially identified as C. diphtheriae 502 but now classified as $C$. belfantii ${ }^{64}$ or C. rouxii ${ }^{65}$, genome-wide average nucleotide 503 identity (ANI) was used as described previously 64. Strains were characterized 504 biochemically for pyrazinamidase, urease, nitrate reductase and for utilization of maltose 505 and trehalose using API Coryne strips (BioMérieux, Marcy l'Etoile, France) and the Rosco 506 Diagnostica reagents (Eurobio, Les Ulis, France). The Hiss serum water test was used 507 for glycogen fermentation. The biovar of isolates was determined based on the 508 combination of nitrate reductase (positive in Mitis and Gravis, negative in Belfanti) and 509 glycogen fermentation (positive in Gravis only). The rare biovar Intermedius was not 510 identified, as its distinction from other biovars is based on colony morphology, which is 511 considered subjective, or on lipophily, which was not tested.

512

\section{Determination of the presence of the tox gene}

514 Determination of the diphtheria toxin gene (tox gene) presence was achieved by a 515 conventional tox PCR assay ${ }^{66}$, while its phenotypic production was assessed by the 516 modified Elek test ${ }^{67}$. We also confirmed tox PCR results by BLASTN (query: tox gene 517 sequence from strain NCTC13129, RefSeq accession number: DIP_RS12515) analysis 518 of the genomic assemblies. 


\section{Antimicrobial susceptibility testing}

521 Phenotypic susceptibility was tested for the following agents: penicillin G (10 IU),

522 amoxicillin, oxacillin, cefotaxime, imipenem, erythromycin, azithromycin, clarithromycin,

523 spiramycin, pristinamycin, kanamycin, gentamicin, rifampicin, tetracycline, ciprofloxacin,

524 clindamycin, sulfonamide, trimethoprim, and trimethoprim + sulfamethoxazole. The 19

525 antimicrobial agents tested (Table S1) corresponded to seven classes, as described

526 hereafter. $\beta$-lactams: penicillin G (PEN), amoxicillin (AMX), oxacillin (OXA), cefotaxime

527 (CFT), imipenem (IMP); Macrolides: azithromycin (AZM), clarithromycin (CLR),

528 erythromycin (ERT) and spiramycin (SPR); Lincosamides: clindamycin (CLD);

529 Streptogramins: pristinamycin (PRT); Aminoglycosides: gentamicin (GEN) and

530 kanamycin (KAN); Folate pathway inhibitors: sulfonamide (SUL), trimethoprim (TMP) and

531 trimethoprim + sulfamethoxazole (cotrimoxazole, TMP-STX); Ansamycins: rifampicin

532 (RIF); Tetracyclines: tetracycline (TET); and Fluoroquinolones: ciprofloxacin (CIP).

533 Antimicrobial susceptibility was determined using the disk diffusion method with

534 impregnated paper disks (Bio-Rad, Marnes-la-Coquette, France) on Mueller Hinton agar

535 plates supplemented with $5 \%$ of sheep blood and $20 \mathrm{mg} / \mathrm{L} \beta-\mathrm{NAD}$, as recommended.

536 Minimum inhibitory concentrations (MIC) were determined using E-test strips

537 (BioMerieux, Marcy l'Etoile, France). The control strain used is S. pneumoniae ATCC

538 49619. The zone diameter (ZD) data were interpreted into S, I and R categories in the

539 following way. First, we used the CA-SFM/EUCAST V.1.0 (Jan 2019) document

540 (https://www.sfm-microbiologie.org/wp-

541 content/uploads/2019/02/CASFM2019 V1.0.pdf), which contains interpretative criteria

542 for Corynebacterium spp. only for CIP, GEN, CLD, TET, RIF and TMP-STX. Second, for

543 the other agents, we used the interpretative criteria published in Table III of the CA-SFM

$544 \quad 2013$

recommendations

545 (https://resapath.anses.fr/resapath uploadfiles/files/Documents/2013 CASFM.pdf).

546 Note that for RIF, we used the 2013 breakpoints, as they fitted better with the observed

547 distribution of ZD values. Clarithromycin breakpoints were taken from those for

548 erythromycin, as recommended. The breakpoint for oxacillin was derived from the one

549 used for Staphylococcus spp. ZD interpretation breakpoints are given in Table S4. 
Penicillin susceptibility was initially determined using $10 \mathrm{UI}$ (6 micrograms) disks

551 (resistance breakpoint: $18 \mathrm{~mm}$ ), but CA-SFM/EUCAST recommendations were changed

552 in 2014 to use $1 \mathrm{UI}$ disks, while the resistance breakpoint was increased from 18 to 29

$553 \mathrm{~mm}$. As all $C$. diphtheriae strains end up in the resistant category following this

554 recommendation, E-test strips were used to define the penicillin MIC since 2014 (Table

555 S1: isolates starting from FRC0259); the EUCAST breakpoint of $0.125 \mathrm{~g} / \mathrm{L}$ was used as

556 cutoff. Penicillin E-test was also performed systematically for strains tested as resistant

557 before 2014 (using $10 \mathrm{Ul}$ disks), as well as for some susceptible isolates (Table S1).

558 Multidrug-resistant C. diphtheriae (MDR-DIP) were defined as strains resistant to

559 more than eight of the agents tested herein, excluding intrinsic resistance to fosfomycin.

560 Note that we used ecological cutoffs rather than currently proposed clinical breakpoints

561 (see Table S5 for a comparison of both types of breakpoints).

562

563

\section{Whole-Genome Sequencing by Illumina and Oxford Nanopore Technologies}

DNA was extracted from broth cultures, by making use of DNeasy Blood \& Tissue Kit

565 (QIAGEN, Hilden, Germany). However, a lysis step was added to the extraction protocol described by the manufacturer as previously described 68: a $1 \mu \mathrm{L}$ loopful of bacterial colonies was emulsified in $180 \mu \mathrm{L}$ of lysis buffer containing $20 \mathrm{mM}$ Tris-HCl, pH8, $2 \mathrm{mM}$ EDTA, $1.2 \%$ Triton X-100, $20 \mathrm{mg} / \mathrm{mL}$ lysozyme, in a DNase/RNase free $1.5 \mathrm{ml}$ Eppendorf tube and incubated in a heating block at $37^{\circ} \mathrm{C}$ for 1 hour, with mixing every 20 min. After extraction, DNA concentration was measured with the Qubit 3.0 Fluorometer (Invitrogen), employing the Qubit dsDNA BR Assay Kit (Invitrogen). Besides, the DNA quality was verified using a D-One spectrophotometer (Nanodrop). Multiplexed paired-end libraries

573 (2 $\times 150$ bp) were prepared using the Nextera XT DNA kit (Illumina, San Diego, CA, USA) 574 and eventually sequenced with an Illumina NextSeq-500 instrument at a minimum of 50X 575 coverage depth. Trimming and clipping were performed using AlienTrimmer v0.4.0 ${ }^{69}$. 576 Redundant or over-represented reads were reduced using the khmer software package $577 \mathrm{~V} 1.3^{70}$. Finally, sequencing errors were corrected using Musket v1.1 ${ }^{71}$. A de novo 578 assembly was performed for each strain using SPAdes v3.12.0 ${ }^{72}$. The genomic 579 sequences of the four reference strains were retrieved from public repositories (Table 580 S1). 
Additionally, the multidrug resistant isolate FRC0402 was subjected to long-read sequencing using Oxford Nanopore Technologies (ONT). Genomic DNA was extracted using the phenol-chloroform protocol combined with Phase Lock Gel tubes (Qiagen

$584 \mathrm{GmbH}$ ). Libraries were prepared using a 1D ligation sequencing kit (SQK-LSK-108) without fragmentation and sequenced using a MinION FLO-MIN-106 flow cell. Finally, ONT and Illumina short reads were combined to generate a hybrid assembly using Unicycler v0.4.4 (normal assembly mode, default parameters).

588

\section{Phylogeny, recombination and genomic sequence analyses}

We built a core genome multiple sequence alignment (cg-MSA) from the assembled genome sequences. For this, the genome sequences were annotated using PROKKA v1.14.2 ${ }^{73}$ with defaults parameters, resulting in GFF files. Roary v3.6 ${ }^{74}$ was used to

593 define protein-coding gene clusters, with a threshold set at $70 \%$ amino acid identity. Core 594 genes were defined as being present in 95\% of genomes and were concatenated into a $595 \mathrm{cg}-\mathrm{MSA}$ by Roary. ClonalFrameML v1.11 ${ }^{75}$ was used to build a phylogenetic tree based on the cg-MSA, which quantifies and accounts for the effects of recombination events. PhyML v20131022 ${ }^{76}$ was used to build an initial tree.

We used Kleborate v1.0.0-beta (https://github.com/katholt/Kleborate), with the -resistance option, to identify (identity $>80 \%$ and coverage $>90 \%$ ) known resistance genes in C. diphtheriae genomic sequences, based on the August 1, 2019 update of the ARGAnnot database. We used BLASTN (identity $>80 \%$ and coverage $>95 \%$ ) to search for the presence of the tox gene and of genes associated with biovar Gravis (DIP351, DIP354 and DIP357) and for nitrate utilization (narKGHJI).

604

MLST genotypes were defined using the international MLST scheme for 606

\section{Genome wide association studies (GWAS)}

The software treeWAS ${ }^{77}$ was used to find genome-wide associations between either antimicrobial resistance phenotypes or biovar on the one hand, and genetic variants (both

610 core-genome SNPs and accessory genome gene presence/absence) on the other hand. 611 Core-genome SNPs were derived either from a mapping approach (Samtools v1.9 and 
612 GATK v3.4-0), which comprises intergenic regions; or from the alignment of core coding 613 sequences found using Roary. We ran treeWAS v1.1 with default parameters, using as 614 input the previously computed ClonalFrameML phylogenetic tree and distribution of 615 homoplasies, in order to account for both the population structure and effect of 616 recombination. For this analysis, susceptibility phenotypes were classified into resistant 617 or susceptible categories based on zone diameter phenotypes using the CA618 SFM/EUCAST 2019 cutoffs (Figure 3, Table S5). The seven chromosomal PBP coding 619 sequences (including gene with locus tag RS14485 in RefSeq NC_002935.2, or DIP0637 620 in the original GenBank file) renamed by us as PBP4b) were extracted from the genomic 621 sequences and translated into amino acid (AA) sequences, which were also analyzed for 622 association with penicillin resistance.

623

624 Cloning and transformation experiments

625 For ectopic expression in C. glutamicum, the pbp2m gene was amplified from 626 C. diphtheriae strain FRC0402 and put under the control of the inducible $P_{\text {gntK }}$ promoter 627 on the shuttle vector pTGR5 ${ }^{78}$ (Figure S10). pbp2m was assembled in this plasmid by 628 Gibson assembly using the primers PBPdi_Fw (CAA AGA AAG GAT AAG ACC ATA TGA 629 TGA CTA AGC ACA ATC GTT TCC GTC), PBPdi_Rv (TAC CTT AAG CGG CCG CTT 630 TAT TGA ATT CCA GAG AAT TTC TGA ACA TCC G), pTGRdi_Fw (TAA AGC GGC 631 CGC TTA AGG TAC C) and pTGRdi_Rv (ATG GTC TTA TCC TTT CTT TGG TGG CG). 632 Escherichia coli CopyCutter EPI400 (Lucigen) was used for cloning of the pbp2m 633 gene and was grown in Luria-Bertani (LB) broth or agar plates at $37^{\circ} \mathrm{C}$ supplemented with $63450 \mu \mathrm{g} / \mathrm{ml}$ kanamycin. The pTGR5_pbp2m plasmid was sequenced and electroporated 635 into Corynebacterium glutamicum ATCC 13032. Positive colonies were grown in brain 636 heart infusion $(\mathrm{BHI})$ at $30^{\circ} \mathrm{C}$ and $120 \mathrm{rpm}$ supplemented with $25 \mu \mathrm{g} / \mathrm{ml}$ kanamycin and $6371 \%(\mathrm{w} / \mathrm{v})$ gluconate when required for ectopic expression of $\mathrm{Pbp} 2 \mathrm{~m}$.

\section{Mapping of SNPs on C. diphtheriae PBP sequences}

Functional annotation of the different sequences was performed with InterPro ${ }^{79}$.

641 Conserved transpeptidation motifs SxxK, SxN and KTG were identified and mapped on 642 the PBP sequences from Corynebacteriales based on the results of multiple sequence 
643 alignments performed with Clustal Omega ${ }^{80}$. When uncertainty between a 644 transmembrane domain and a signal peptide existed, a decision was made based on 645 previous characterization of the homologous PBP in other Corynebacteriales in the 646 literature.

647

\section{Data availability}

649 The genomic sequencing data generated in this study were deposited in the European 650 Nucleotide Archive (ENA) database and are accessible through the BioProject 651 PRJEB22103.

652

\section{Code availability}

654 No new code was used to analyse the findings in this study.

655 
656

657

658

659

660

661

662

663

664

665

666

667

668

669

670

671

672

673

674

675

676

677

678

679

\section{REFERENCES}

1. Patey, O. et al. Clinical and molecular study of Corynebacterium diphtheriae systemic infections in France. Coryne Study Group. J. Clin. Microbiol. 35, 441-445 (1997).

2. Hadfield, T. L., McEvoy, P., Polotsky, Y., Tzinserling, V. A. \& Yakovlev, A. A. The pathology of diphtheria. J. Infect. Dis. 181 Suppl 1, S116-120 (2000).

3. Burkovski, A. Corynebacterium diphtheriae and Related Toxigenic Species: Genomics, Pathogenicity and Applications. (Springer, 2014).

4. Sharma, N. C. et al. Diphtheria. Nat. Rev. Dis. Primer 5, 81 (2019).

5. Freeman, V. J. Studies on the virulence of bacteriophage-infected strains of Corynebacterium diphtheriae. J. Bacteriol. 61, 675-688 (1951).

6. Parveen, S., Bishai, W. R. \& Murphy, J. R. Corynebacterium diphtheriae: Diphtheria Toxin, the tox Operon, and Its Regulation by Fe2+ Activation of apo-DtxR. Microbiol. Spectr. 7, (2019).

7. Pappenheimer, A. M. \& Murphy, J. R. Studies on the molecular epidemiology of diphtheria. Lancet Lond. Engl. 2, 923-926 (1983).

8. Trost, E. et al. Pangenomic study of Corynebacterium diphtheriae that provides insights into the genomic diversity of pathogenic isolates from cases of classical diphtheria, endocarditis, and pneumonia. J. Bacteriol. 194, 3199-3215 (2012).

9. Sangal, V. \& Hoskisson, P. A. Evolution, epidemiology and diversity of Corynebacterium diphtheriae: New perspectives on an old foe. Infect. Genet. Evol. J. Mol. Epidemiol. Evol. Genet. Infect. Dis. 43, 364-370 (2016). 
680

681

682

683

684

685

686

687

688

689

690

691

692

693

694

695

696

697

698

699

700

701

702

10. Timms, V. J., Nguyen, T., Crighton, T., Yuen, M. \& Sintchenko, V. Genome-wide comparison of Corynebacterium diphtheriae isolates from Australia identifies differences in the Pan-genomes between respiratory and cutaneous strains. $B M C$ Genomics 19, 869 (2018).

11. Sangal, V. et al. A lack of genetic basis for biovar differentiation in clinically important Corynebacterium diphtheriae from whole genome sequencing. Infect. Genet. Evol. J. Mol. Epidemiol. Evol. Genet. Infect. Dis. 21, 54-57 (2014).

12. Bolt, F. et al. Multilocus sequence typing identifies evidence for recombination and two distinct lineages of Corynebacterium diphtheriae. J. Clin. Microbiol. 48, 4177-4185 (2010).

13. Grosse-Kock, S. et al. Genomic analysis of endemic clones of toxigenic and non-toxigenic Corynebacterium diphtheriae in Belarus during and after the major epidemic in 1990s. BMC Genomics 18, 873 (2017).

14. Dittmann, S. et al. Successful control of epidemic diphtheria in the states of the Former Union of Soviet Socialist Republics: lessons learned. J. Infect. Dis. 181 Suppl 1, S10-22 (2000).

15. WHO. Surveillance and burden of diphtheria. http://www.who.int/immunization/monitoring_surveillance/burden/diphtheria/en/. (2018).

16. Galazka, A. The changing epidemiology of diphtheria in the vaccine era. J. Infect. Dis. 181 Suppl 1, S2-9 (2000).

17. Rahman, M. R. \& Islam, K. Massive diphtheria outbreak among Rohingya refugees: lessons learnt. J. Travel Med. 26, (2019). 
703

704

705

706

707

708

709

710

711

712

713

714

715

716

717

718

719

720

721

722

723

724

725

18. Dureab, F., Müller, O. \& Jahn, A. Resurgence of diphtheria in Yemen due to population movement. J. Travel Med. 25, (2018).

19. Zakikhany, K. \& Efstratiou, A. Diphtheria in Europe: current problems and new challenges. Future Microbiol. 7, 595-607 (2012).

20. Meinel, D. M. et al. Outbreak investigation for toxigenic Corynebacterium diphtheriae wound infections in refugees from Northeast Africa and Syria in Switzerland and Germany by whole genome sequencing. Clin. Microbiol. Infect. Off. Publ. Eur. Soc. Clin. Microbiol. Infect. Dis. 22, 1003.e1-1003.e8 (2016).

21. Scheifer, C. et al. Re-emergence of Corynebacterium diphtheriae. Med. Mal. Infect. 49, 463-466 (2019).

22. Möller, J. et al. Proteomics of diphtheria toxoid vaccines reveals multiple proteins that are immunogenic and may contribute to protection of humans against Corynebacterium diphtheriae. Vaccine 37, 3061-3070 (2019).

23. Zasada, A. A. Antimicrobial susceptibility and treatment. in Corynebacterium diphtheriae and related toxigenic species genomics, pathogenicity and applications. Burkovski A., editor. 239-246 (New York: Springer, 2014).

24. Kupferschmidt, K. Life-saving diphtheria drug is running out. Science 355, 118-119 (2017).

25. PAHO/WHO, P. A. H. O. / W. H. O. Epidemiological Update: Diphtheria. (2018).

26. Kneen, R. et al. Penicillin vs. erythromycin in the treatment of diphtheria. Clin. Infect. Dis. Off. Publ. Infect. Dis. Soc. Am. 27, 845-850 (1998).

27. Pereira, G. A. et al. Antimicrobial resistance among Brazilian Corynebacterium diphtheriae strains. Mem. Inst. Oswaldo Cruz 103, 507-510 (2008). 
726 28. von Hunolstein, C., Scopetti, F., Efstratiou, A. \& Engler, K. Penicillin tolerance amongst non-toxigenic Corynebacterium diphtheriae isolated from cases of pharyngitis. J.

728 Antimicrob. Chemother. 50, 125-128 (2002).

29. Benamrouche, N. et al. Microbiological and molecular characterization of Corynebacterium diphtheriae isolated in Algeria between 1992 and 2015. Clin. Microbiol. Infect. Off. Publ. Eur. Soc. Clin. Microbiol. Infect. Dis. 22, 1005.e1-1005.e7 (2016).

30. Paveenkittiporn, W., Sripakdee, S., Koobkratok, O., Sangkitporn, S. \& Kerdsin, A. Molecular epidemiology and antimicrobial susceptibility of outbreak-associated Corynebacterium diphtheriae in Thailand, 2012. Infect. Genet. Evol. J. Mol. Epidemiol. Evol. Genet. Infect. Dis. 75, 104007 (2019).

31. Husada, D. et al. First-line antibiotic susceptibility pattern of toxigenic Corynebacterium diphtheriae in Indonesia. BMC Infect. Dis. 19, 1049 (2019).

32. Bernard, K. \& Pacheco, A. L. In Vitro Activity of 22 Antimicrobial Agents against Corynebacterium and Microbacterium Species Referred to the Canadian National Microbiology Laboratory. Clin. Microbiol. Newsl. 37, 187-198 (2015). diphtheroids mediating erythromycin resistance. Antimicrob. Agents Chemother. 18, 814-821 (1980). and lincomycin. Lancet Lond. Engl. 1, 156 (1973). diphtheriae. J. Antimicrob. Chemother. 36, 1108-1110 (1995). 
36. Mina, N. V. et al. Canada's first case of a multidrug-resistant Corynebacterium diphtheriae strain, isolated from a skin abscess. J. Clin. Microbiol. 49, 4003-4005

751 (2011).

37. Tauch, A., Bischoff, N., Brune, I. \& Kalinowski, J. Insights into the genetic organization of the Corynebacterium diphtheriae erythromycin resistance plasmid pNG2 deduced from its complete nucleotide sequence. Plasmid 49, 63-74 (2003).

38. Barraud, O., Badell, E., Denis, F., Guiso, N. \& Ploy, M.-C. Antimicrobial drug resistance in Corynebacterium diphtheriae mitis. Emerg. Infect. Dis. 17, 2078-2080 (2011).

39. Cerdeño-Tárraga, A. M. et al. The complete genome sequence and analysis of Corynebacterium diphtheriae NCTC13129. Nucleic Acids Res. 31, 6516-6523 (2003).

40. Grimont, P. A. D. et al. International nomenclature for Corynebacterium diphtheriae ribotypes. Res. Microbiol. 155, 162-166 (2004).

41. Santos, A. S. et al. Searching whole genome sequences for biochemical identification features of emerging and reemerging pathogenic Corynebacterium species. Funct. Integr. Genomics 18, 593-610 (2018).

42. Tagini, F. et al. Distinct Genomic Features Characterize Two Clades of Corynebacterium diphtheriae: Proposal of Corynebacterium diphtheriae Subsp. diphtheriae Subsp. nov. and Corynebacterium diphtheriae Subsp. lausannense Subsp. nov. Front. Microbiol. 9, 1743 (2018).

43. Kahlmeter, G. et al. European harmonization of MIC breakpoints for antimicrobial susceptibility testing of bacteria. J. Antimicrob. Chemother. 52, 145-148 (2003). 
770

771

772

773

774

775

776

777

778

779

780

781

782

783

784

785

786

787

788

789

790

791

792

44. Tauch, A., Kassing, F., Kalinowski, J. \& Pühler, A. The Corynebacterium xerosis composite transposon Tn5432 consists of two identical insertion sequences, designated IS1249, flanking the erythromycin resistance gene ermCX. Plasmid 34, 119-131 (1995).

45. Lavollay, M. et al. The beta-lactam-sensitive D,D-carboxypeptidase activity of Pbp4 controls the L,D and D,D transpeptidation pathways in Corynebacterium jeikeium. Mol. Microbiol. 74, 650-661 (2009).

46. Seth-Smith, H. M. B. \& Egli, A. Whole Genome Sequencing for Surveillance of Diphtheria in Low Incidence Settings. Front. Public Health 7, 235 (2019).

47. Barksdale, L. Corynebacterium diphtheriae and its relatives. Bacteriol. Rev. 34, 378-422 (1970).

48. McLeod, J. W. THE TYPES MITIS, INTERMEDIUS AND GRAVIS OF CORYNEBACTERIUM DIPHTHERIAE: A Review of Observations during the Past Ten Years. Bacteriol. Rev. 7, $1-41$ (1943).

49. Zou, J. et al. Phenotypic and Genotypic Correlates of Penicillin Susceptibility in Nontoxigenic Corynebacterium diphtheriae, British Columbia, Canada, 2015-2018. Emerg. Infect. Dis. 26, 97-103 (2020).

50. Sariadji, K., Puspandari, S. N. \& Sembiring, M. Antibiotic Susceptibility Pattern of Corynebacterium diphtheriae Isolated from Outbreaks in Indonesia 2010-2015. Indones Biomed J 10, 51-55 (2018).

51. Parande, M. V., Roy, S., Mantur, B. G., Parande, A. M. \& Shinde, R. S. Resurgence of diphtheria in rural areas of North Karnataka, India. Indian J. Med. Microbiol. 35, 247251 (2017).

52. Anonymous. Conduite à tenir lors de l'apparition d'un cas de diphtérie. (2011). 
793 53. Tauch, A., Götker, S., Pühler, A., Kalinowski, J. \& Thierbach, G. The 27.8-kb R-plasmid

794 pTET3 from Corynebacterium glutamicum encodes the aminoglycoside

795 adenyltransferase gene cassette aadA9 and the regulated tetracycline efflux system Tet

79633 flanked by active copies of the widespread insertion sequence IS6100. Plasmid 48,

$797 \quad 117-129(2002)$.

798 54. Tauch, A., Kassing, F., Kalinowski, J. \& Pühler, A. The erythromycin resistance gene of

799 the Corynebacterium xerosis R-plasmid pTP10 also carrying chloramphenicol,

800 kanamycin, and tetracycline resistances is capable of transposition in Corynebacterium

801 glutamicum. Plasmid 33, 168-179 (1995).

802 55. Yoon, S., Kim, H., Lee, Y. \& Kim, S. Bacteremia caused by Corynebacterium amycolatum

803 with a novel mutation in gyrA gene that confers high-level quinolone resistance. Korean

804 J. Lab. Med. 31, 47-48 (2011).

805 56. Sierra, J. M., Martinez-Martinez, L., Vázquez, F., Giralt, E. \& Vila, J. Relationship between

806 mutations in the gyrA gene and quinolone resistance in clinical isolates of

807 Corynebacterium striatum and Corynebacterium amycolatum. Antimicrob. Agents

808 Chemother. 49, 1714-1719 (2005).

809 57. Pivot, D. et al. Carriage of a single strain of non-toxigenic Corynebacterium diphtheriae

810 biovar Belfanti (Corynebacterium belfantii) in four patients with cystic fibrosis. J. Clin.

811 Microbiol. (2019) doi:10.1128/JCM.00042-19.

812 58. Fishovitz, J. et al. Disruption of allosteric response as an unprecedented mechanism of

813 resistance to antibiotics. J. Am. Chem. Soc. 136, 9814-9817 (2014). 
814

815

816

817

818

819

820

821

822

823

824

825

826

827

828

829

830

831

832

833

834

835

59. Otero, L. H. et al. How allosteric control of Staphylococcus aureus penicillin binding protein 2 a enables methicillin resistance and physiological function. Proc. Natl. Acad. Sci. U. S. A. 110, 16808-16813 (2013).

60. Bernardo-García, N. et al. Allostery, Recognition of Nascent Peptidoglycan, and Crosslinking of the Cell Wall by the Essential Penicillin-Binding Protein 2x of Streptococcus pneumoniae. ACS Chem. Biol. 13, 694-702 (2018).

61. Chewapreecha, C. et al. Comprehensive identification of single nucleotide polymorphisms associated with beta-lactam resistance within pneumococcal mosaic genes. PLoS Genet. 10, e1004547 (2014).

62. Farfour, E. et al. Characterization and comparison of invasive Corynebacterium diphtheriae isolates from France and Poland. J. Clin. Microbiol. 50, 173-175 (2012).

63. Park, W. H. \& Williams, A. W. The production of diphtheria toxin. J. Exp. Med. 1, 164-185 (1896).

64. Dazas, M., Badell, E., Carmi-Leroy, A., Criscuolo, A. \& Brisse, S. Taxonomic status of Corynebacterium diphtheriae biovar Belfanti and proposal of Corynebacterium belfantii sp. nov. Int. J. Syst. Evol. Microbiol. 68, 3826-3831 (2018).

65. Badell, E. et al. Corynebacterium rouxii sp. nov., a novel member of the diphtheriae species complex. Res. Microbiol. (2020) doi:10.1016/j.resmic.2020.02.003.

66. Hauser, D., Popoff, M. R., Kiredjian, M., Boquet, P. \& Bimet, F. Polymerase chain reaction assay for diagnosis of potentially toxinogenic Corynebacterium diphtheriae strains: correlation with ADP-ribosylation activity assay. J. Clin. Microbiol. 31, 2720-2723 (1993). 
836

837

838

839

840

841

842

843

844

845

846

847

848

849

850

851

852

853

854

855

856

857

67. Engler, K. H., Glushkevich, T., Mazurova, I. K., George, R. C. \& Efstratiou, A. A modified Elek test for detection of toxigenic corynebacteria in the diagnostic laboratory. J. Clin. Microbiol. 35, 495-498 (1997).

68. Badell, E. et al. Improved quadruplex real-time PCR assay for the diagnosis of diphtheria. J. Med. Microbiol. 68, 1455-1465 (2019).

69. Criscuolo, A. \& Brisse, S. AlienTrimmer: A tool to quickly and accurately trim off multiple short contaminant sequences from high-throughput sequencing reads. Genomics 10.1016/j.ygeno.2013.07.011 (2013) doi:10.1016/j.ygeno.2013.07.011.

70. Crusoe, M. R. et al. The khmer software package: enabling efficient nucleotide sequence analysis. F1000Research 4, 900 (2015).

71. Liu, Y., Schröder, J. \& Schmidt, B. Musket: a multistage k-mer spectrum-based error corrector for Illumina sequence data. Bioinforma. Oxf. Engl. 29, 308-315 (2013).

72. Bankevich, A. et al. SPAdes: a new genome assembly algorithm and its applications to single-cell sequencing. J Comput Biol 19, 455-77 (2012).

73. Seemann, T. Prokka: rapid prokaryotic genome annotation. Bioinforma. Oxf. Engl. 30, 2068-2069 (2014).

74. Page, A. J. et al. Roary: rapid large-scale prokaryote pan genome analysis. Bioinforma. Oxf. Engl. 31, 3691-3693 (2015).

75. Didelot, X. \& Wilson, D. J. ClonalFrameML: efficient inference of recombination in whole bacterial genomes. PLoS Comput. Biol. 11, e1004041 (2015).

76. Guindon, S. et al. New algorithms and methods to estimate maximum-likelihood phylogenies: assessing the performance of PhyML 3.0. Syst. Biol. 59, 307-321 (2010). 
858 77. Collins, C. \& Didelot, X. A phylogenetic method to perform genome-wide association

859 studies in microbes that accounts for population structure and recombination. PLoS

860 Comput. Biol. 14, e1005958 (2018).

861 78. Sogues, A. et al. Essential dynamic interdependence of FtsZ and SepF for Z-ring and

862 septum formation in Corynebacterium glutamicum. Nat. Commun. 11, 1641 (2020).

863 79. Mitchell, A. L. et al. InterPro in 2019: improving coverage, classification and access to

864 protein sequence annotations. Nucleic Acids Res. 47, D351-D360 (2019).

865 80. Madeira, F. et al. The EMBL-EBI search and sequence analysis tools APIs in 2019.

$866 \quad$ Nucleic Acids Res. 47, W636-W641 (2019).

867 
868

869

870

871

872

873

874

875

876

877

878

879

880

881

882

883

884

885

886

887

888

889

890

891

892

893

894

895

896

897

\section{Acknowledgements}

We thank Vincent Enouf and the P2M core facility of Institut Pasteur for genomic sequencing. We are indebted to Collection de l'Institut Pasteur for providing reference strains of ribotypes, which were deposited following their described by Grimont and colleagues in 2004 40. We thank Valerie Bouchez for help with Oxford Nanopore Technologies sequencing.

\section{Author contributions}

S.B. conceived, designed and coordinated the study. A.C-L., E.B., M.B. and M.D. performed the microbiological cultures of the isolates and their biochemical and molecular characterizations. M.H., L.G.P., C.R., S.L.B., M.B.-P., M.D. and S.B. analysed the genomic and phenotypic data. J.T. reviewed the clinical source data of the isolates. Q.G. and A.-M.W. performed the pbp2m cloning experiments and analyzed the PBP sequences. X.D. provided help with the phylogenetic, recombination and GWAS analyses. S.B. wrote the initial version of the manuscript. All authors provided input to the manuscript and reviewed the final version.

\section{Competing interests}

The authors declare no competing interests.

\section{Funding}

$\mathrm{MH}$ was supported financially by a PhD grant from the European Joint Programme One Health, which has received funding from the European Union's Horizon 2020 Research and Innovation Programme under Grant Agreement No. 773830. LGP was supported financially by a grant from the Institut Français de Bioinformatique, the national infrastructure for services in bioinformatics created in the frame of the French Government's Investissement d'Avenir program. QG was funded by MTCI PhD school (ED 563). CR was supported by a Pasteur-Roux fellowship from Institut Pasteur. S.L.B. received support from a Victorian Fellowship, provided by VESKI and funded by the State Government of Victoria, Australia. 
898 The National Reference Center for Corynebacteria of the diphtheriae complex 899 receives support from Institut Pasteur and Public Health France (Santé Publique France, 900 Saint Maurice, France). This work was supported financially by the French Government's 901 Investissement d'Avenir program Laboratoire d'Excellence "Integrative Biology of 902 Emerging Infectious Diseases" (ANR-10-LABX-62-IBEID). AW and QG receive support 903 from Institut Pasteur and the CNRS (France).

904

905 
Figure 1. Temporal and geographical distribution of strains studied. represented in red or yellow shaded patterns (see key), whereas the older clinical isolates are in grey and the reference strains in blue. The inset shows pie charts with the frequency

912 of resistance phenotypes among strains from the four most represented geographic 913 origins; the remaining ones are pooled in the right-most pie chart. B. Geographic origins

914 of strains from the three subsets.

915

\section{Figure 2. Phylogenetic tree of $C$. diphtheriae}

917 The tree was obtained using ClonalFrameML and was rooted using C. rouxii and 918 C. belfantii isolates (not shown). Main lineages Mitis and Gravis are labeled and their

919 branches are drawn using purple and green, respectively. The first (internal) circle around 920 the tree corresponds to the three strain subsets (red: recent clinical isolates; blue: 921 reference strains; grey: older clinical isolates). The second circle (stars) gives the 922 toxigenic status. The third circle corresponds to biovars Mitis (purple), Gravis (green) and 923 Belfanti (yellow). The next three circles indicate the presence of the spuA-associated 924 gene cluster; DIP357 = spuA gene. The positions of reference strains PW8, NCTC13129 925 and NCTC10648 are indicated. The scale bar give the number of nucleotide substitutions 926 per site.

927

928 Figure 3. The distributions of zone diameter values for 19 antimicrobial agents

929 A: beta-lactams, macrolides and pristinamycin. B: other agents. X-axis: diameter in mm; 930 Y-axis: number of strains. Colors inside the bars represent subset and geographic origins 931 as in Figure 1 (see key on panel B). The three background colors represent the 932 categorical interpretations according to EUCAST: resistant (salmon, left), intermediate 933 (lighter salmon, middle) and susceptible (pale beige, right). The grey vertical bar 934 corresponds to the proposed tentative ecological cutoff. 
936 Figure 4. Proportions of resistant strains by antimicrobial agent and multidrug 937 resistance phenotypes.

938 Interpretation of zone diameter values was performed according to proposed ecological 939 cutoffs. The main panel shows the percentage of strains resistant to each agent. Tmp940 stx: trimethoprim-sulfamethoxazole. The four bars for each agent correspond to the entire 941 dataset (all, shaded green) or the three subsets (see key). The inset shows the number

942 of strains resistant to a given number of antimicrobials. Penicillin and/or erythromycin 943 resistant strains are colored with darker grey (see key). The vertical bar indicates the 944 definition of multidrug resistant isolates (> 8 agents). FRC0402, the most multidrug 945 resistant isolate with resistance to 12 agents, is highlighted.

946

947 Figure 5. Phylogenetic distribution of antimicrobial resistance phenotypes and 948 genes

949 The phylogenetic tree is the same as in Figure 1; the Mitis branch is in purple, the Gravis 950 branch in green. To the right of the tree, each bloc indicates first, the phenotype (resistant: 951 red; see key) and relevant corresponding genotypes (orange: gene or mutation 952 presence). The last bloc shows resistance genes linked to chloramphenicol, which was 953 not tested phenotypically.

954

955 Figure 6. Phenotypic effect of pbp2m expression

956 Compared susceptibility phenotypes for C. glutamicum transformants with plasmid 957 pTGR5 containing, or not, the pbp2m gene. Left, shift in zone diameter size; right, shift 958 in the minimum inhibitory concentration (MIC). Diamonds are positioned on the scales, at 959 positions corresponding to the difference of zone diameters (without pbp2m - with $960 \mathrm{pbp} 2 \mathrm{~m}$ ) or the log2 of $\mathrm{MIC}$ ratios (with $p b p 2 m /$ without $p b p 2 m$ ). Red, penicillins or 961 cephalosporins; blue, other agents. Tmp-Stx: trimethoprim-sulfamethoxazole.

962

963 Figure 7. Map of plasmid pLRPD from isolate FRC0402

964 Predicted coding sequences are portrayed by arrows and coloured based on the 965 predicted gene function (refer to key). Inner blue circle, $\mathrm{G}+\mathrm{C} \%$ content; inner green 966 circle, A+T\% content. IS3502 annotation is putative; IS3503 is truncated. 


\section{Supplementary Figures}

968

969 Figure S1. Biovar and tox status of the three strain subsets

970 In the upper panel, the numbers of strains are given separately for the three subsets of

971 strains; the recent clinical isolates one (right hand side) is broken down by individual year.

972 Colors correspond to biovars (see key) and shaded areas denote tox-positive isolates. In 973 the lower panel, the percentage of tox-positive strains (red bars) and of tox-positive or 974 tox-negative strains per biovar (see key), are given for the entire dataset and for the three 975 subsets separately; shaded sectors correspond to tox-positive strains within each biovar. 976

977 Figure S2. Phylogenetic tree of $\boldsymbol{C}$. diphtheriae, with isolates names

978 The phylogenetic tree and outer information correspond to those in Figure 2, with the 979 addition of isolates names, geographic origins and year of isolation.

980

981

982

Figure S3. Genomic difference between reference strains PW8 (biovar Mitis) and

983 NCTC13129 (Gravis)

984

The genomic region of approx. $10 \mathrm{~kb}$ inserted in biovar Gravis strain NCTC13129 includes

985 genes DIP0351, DIP0354 and spuA (DIP0357); these three accessory genes are strongly associated with biovar Gravis, as is the SNP at position 324,487.

986

987

988

Figure S4. The distributions of zone diameter values for 19 antimicrobial agents, colored by the presence of the tox gene

989

A: beta-lactams, macrolides and pristinamycin. B: other agents. X-axis: diameter in $\mathrm{mm}$;

990 Y-axis: number of strains. Colors inside the bars represent tox-positive isolates (red) or

991 tox-negative isolates (grey). The three background colors represent the categorical 992 interpretations according to EUCAST: resistant (salmon, left), intermediate (lighter 993 salmon, middle) and susceptible (pale beige, right). The grey vertical bar corresponds to 994 the proposed tentative ecological cutoff.

995

996 Figure S5. The distributions of zone diameter values for 19 antimicrobial agents, 997 colored by the main lineages (Mitis and Gravis) 
998

999

1000

1001

1002

1003

1004

1005

1006

1007

1008

1009

1010

1011

1012

1013

1014

1015

1016

1017

1018

1019

1020

1021

1022

1023

1024

1025

1026

1027

1028

A: beta-lactams, macrolides and pristinamycin. B: other agents. X-axis: diameter in mm; Y-axis: number of strains. Colors inside the bars represent the two main lineages (see key in panel B). The three background colors represent the categorical interpretations according to EUCAST: resistant (salmon, left), intermediate (lighter salmon, middle) and susceptible (pale beige, right). The grey vertical bar corresponds to the proposed tentative ecological cutoff.

\section{Figure S6. Correlation plot of antimicrobial resistance phenotypes and genotypes.}

The correlation matrix between antimicrobial resistance genotype and phenotype is based on the correlation for binary variables (in the case of resistance genes: 1, presence; 0 , absence; in the case of antimicrobial drugs: 1 , resistant/intermediate; 0 , susceptible) using the 'corr.test' function (Pearson method, which for a pair of binary variables equates to the Phi coefficient) from the 'corrplot' R package. Significant correlations were visualized utilizing the 'corrplot' function from the same package. Blank squares represent correlations without statistical significance ( $p>0.05$ ). Positive correlation is depicted by blue circles, whereas red circles represent significant negative correlation. The size and strength of color represent the numerical value of the Phi correlation coefficient. Black rectangles group genes commonly found together in the same strain. Genes cmx and cmIA5 are known to be associated with chloramphenicol resistance, which was not tested here.

Figure S7. treeWAS results plots for ciprofloxacin, rifampicin and trimethoprim

Distribution of treeWAS scores obtained for genome-wide SNPs in association with ciprofloxacin, rifampicin and trimethoprim. Significant SNPs in $g y r A$, rpoB and folA are indicated.

Figure S8. treeWAS results for amino acid polymorphisms in the chromosomal PBP coding genes of $C$. diphtheriae

Statistical significance of the treeWAS subsequent score obtained when testing the association of deduced amino-acid alterations in the seven chromosomal PBP sequences, and penicillin resistance phenotype. Within each of the seven panel the X- 
1029 axis represent the amino acid sequence (numbers: AA positions), and the Y-axis the $1030 \log 10$ (p-value). The positions of transglycosylase, transpeptidase, carboxypeptidase or 1031 other relevant domains of the PBP are shaded in grey. SNPs are represented as blue or 1032 orange circles (in alternance) at their corresponding position. The red bar indicates the 10330.05 p-value position. The most significant SNP, at position 535 of PBP2b, is circled.

1035 Figure S9. Functional annotation and mapping of significant SNPs associated with 1036 C. diphtheriae chromosomal PBPs.

1037 Conserved transpeptidation motifs SxxK SxN KTG are indicated on the transpeptidase 1038 and carboxypeptidase domains by white lines. Significant SNPs associated with penicillin 1039 resistance are indicted by red pins. The PBPs correspond to the following genes: pbp1a 1040 (DIP2294), pbp1b (DIP0298), pbp2a (DIP0055), pbp2b (DIP1604), pbp2c (DIP1497), 1041 pbp4 (DIP2005) and pbp4b (RS14485 = DIP0637). Pbp2m was not analyzed for amino1042 acid changes associated with penicillin resistance, as it corresponds to an accessory 1043 PBP.

1044

1045 Figure S10. Construction strategy of plasmid pTGR5_pbp2m

1046 The pbp2m gene was PCR amplified and combined with plasmid pTGR5 using Gibson 1047 assembly as indicated.

1048

1049 Figure S11. Genetic context of the pbp2m gene in Corynebacterium

1050 The genomic context of pbp2m in C. diphtheriae and other Corynebacterium strains that 1051 possess this gene is given for representative genomes of the diversity that was found. 1052 Genes pbp2m, blaB and lys $R$ are represented with a dark red background; these three 1053 genes were always associated and constitute the pbp-containing unit (PCU). Gene ermX 1054 is in yellow. A putative helicase often associated with the PCU is represented in pink; a 1055 relaxase gene is shaded in green. Black arrows represent insertion sequence genes. Six 1056 groups were defined based on conserved features, as indicated. Dark grey parallelepiped 1057 joining different genomes represent homology levels, as indicated in the gradient key. 1058 Strains of the present study with identical structures as those represented are indicated 
bioRxiv preprint doi: https://doi.org/10.1101/2020.05.19.101030; this version posted May 20, 2020. The copyright holder for this preprint (which

was not certified by peer review) is the author/funder, who has granted bioRxiv a license to display the preprint in perpetuity. It is made available under aCC-BY-NC-ND 4.0 International license.

1059 in parentheses below the strain name of the representative genome. The scale bar 1060 represents $12 \mathrm{~kb}$. 


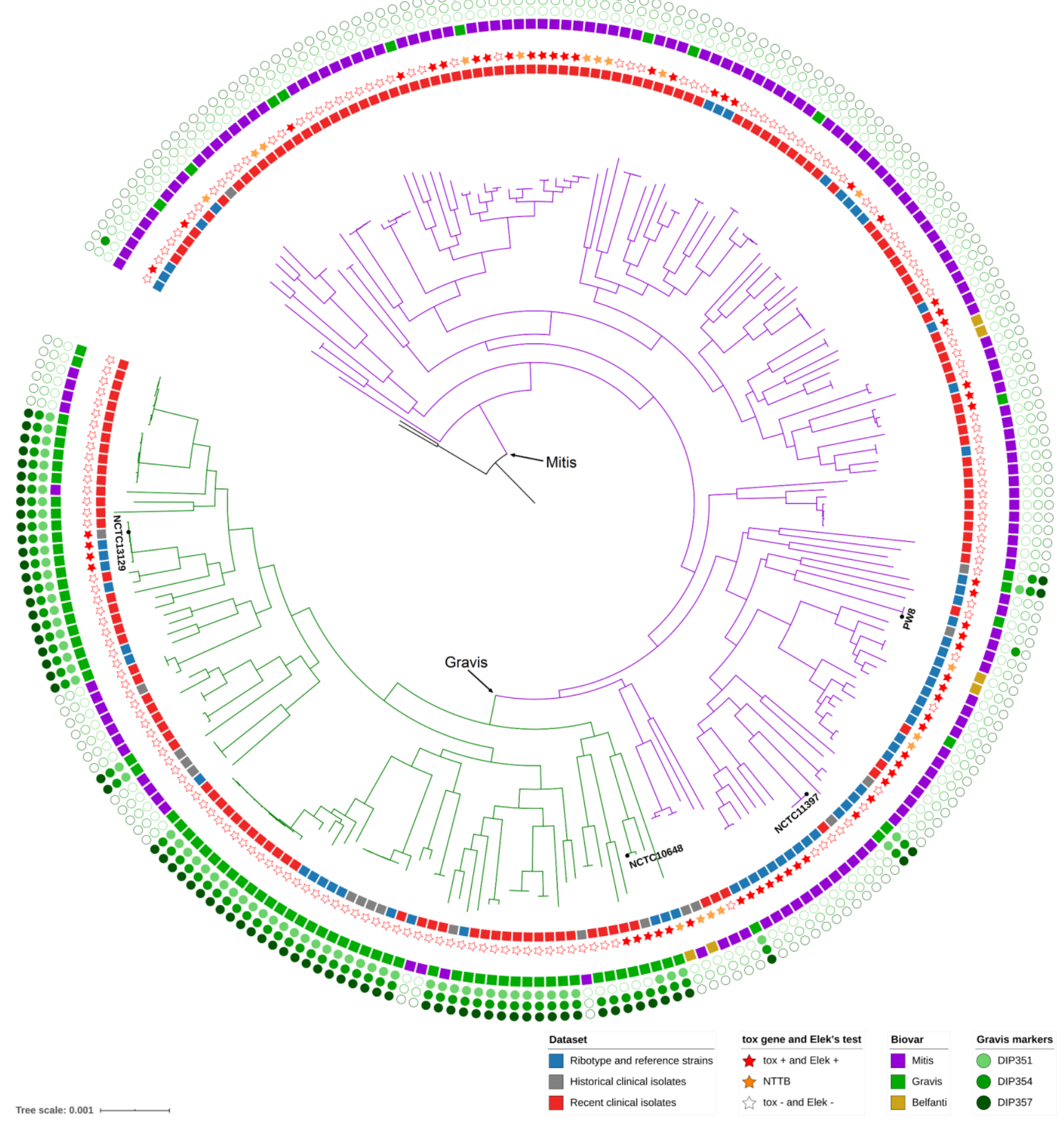


(A)
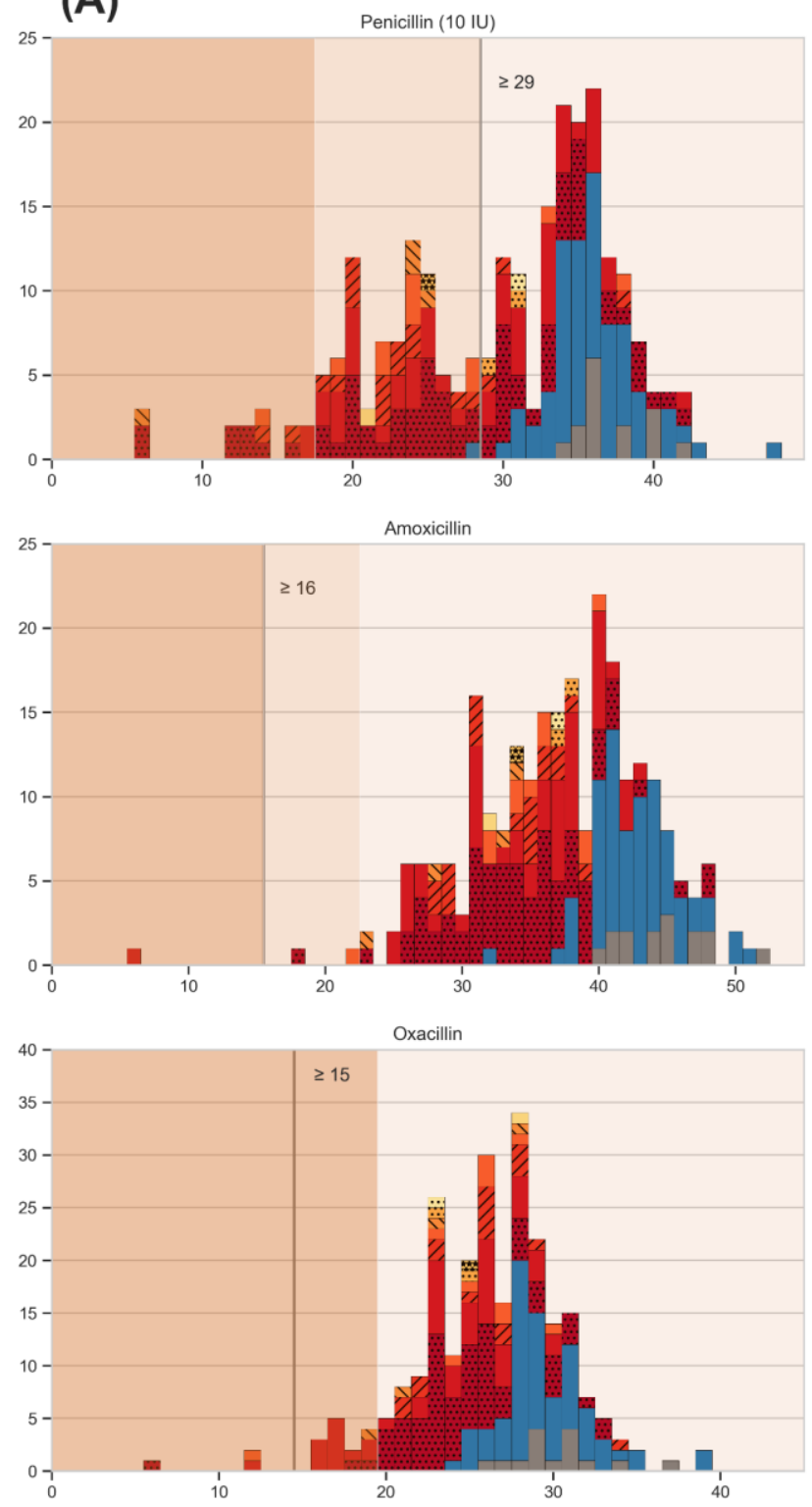

Oxacillin

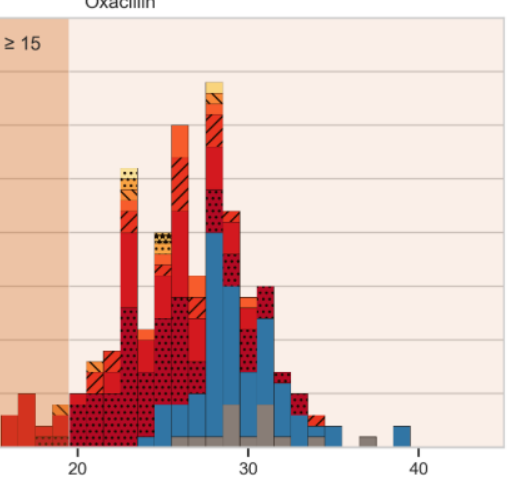

Cefotaxime
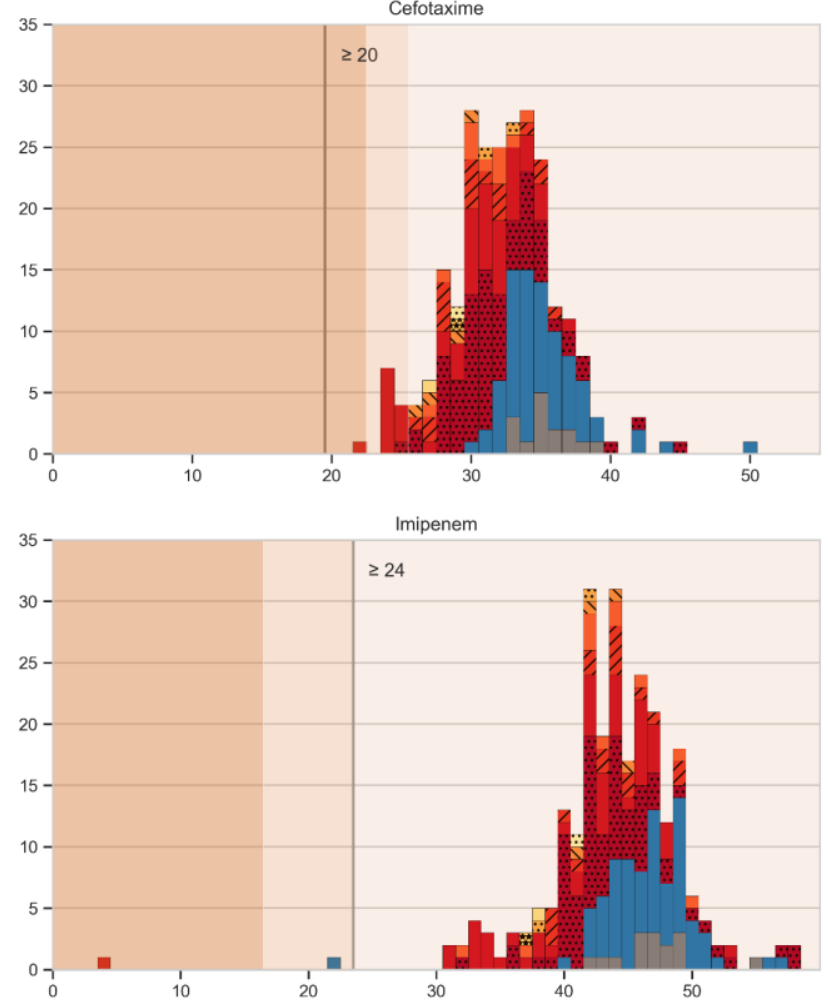

has granted bioRxiv a license to display the preprint in perpetuity. It is made -BY-NC-ND 4.0 International license.
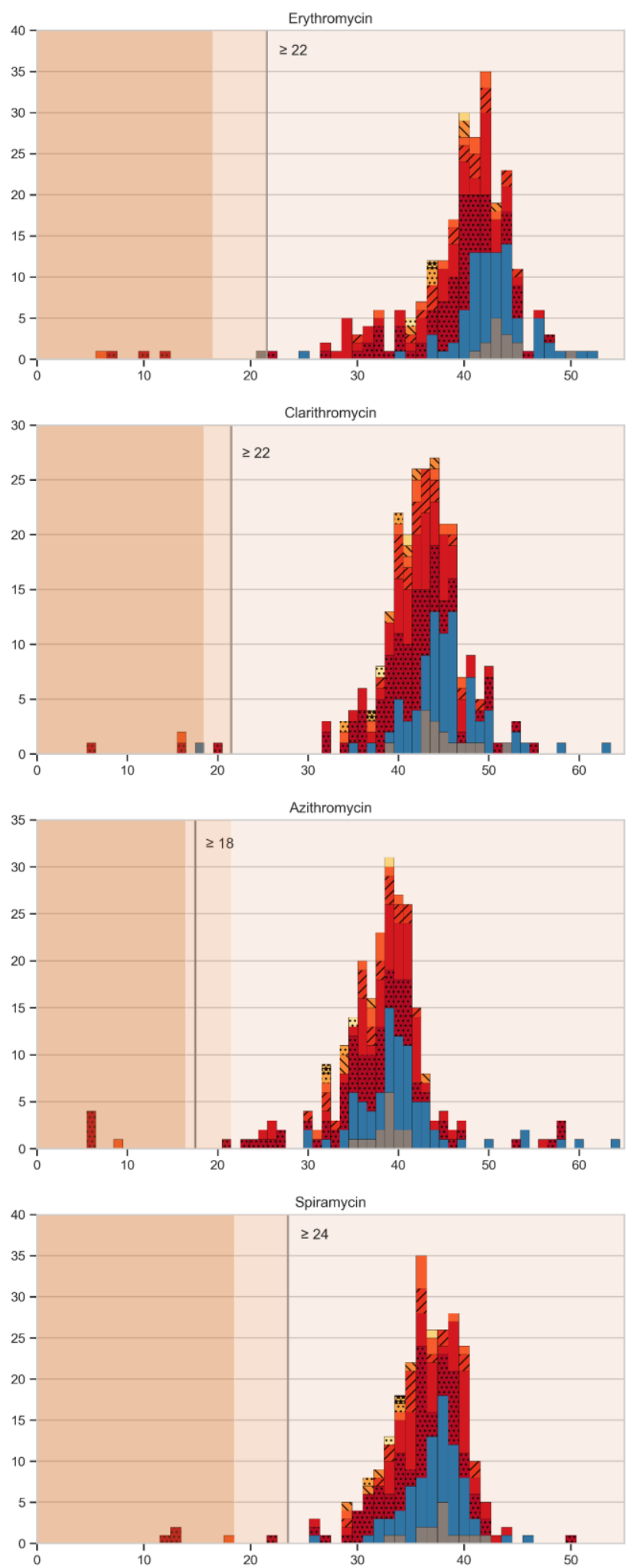

Pristinamycin

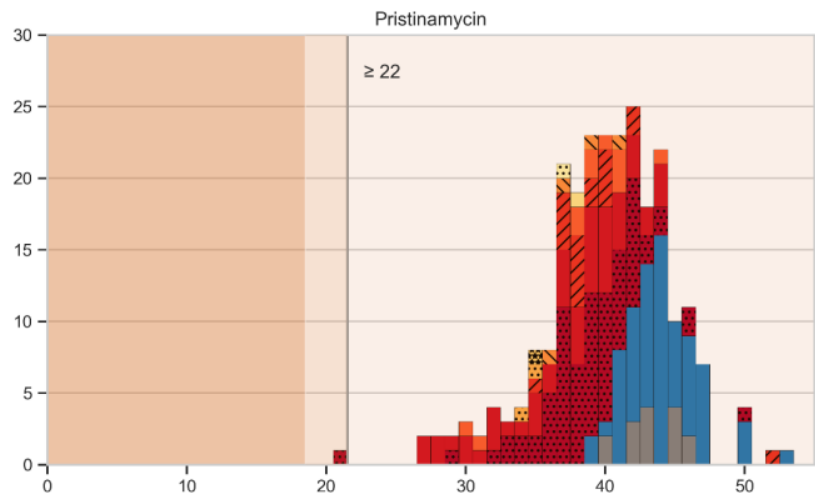


(B)

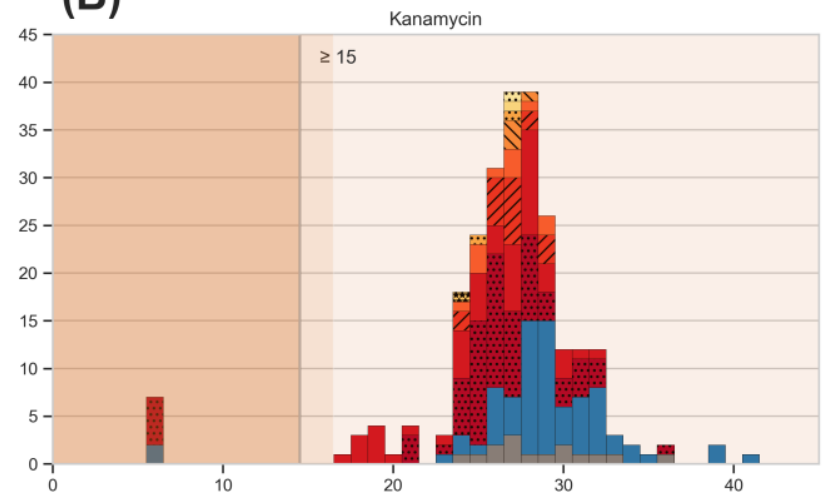

Gentamicin
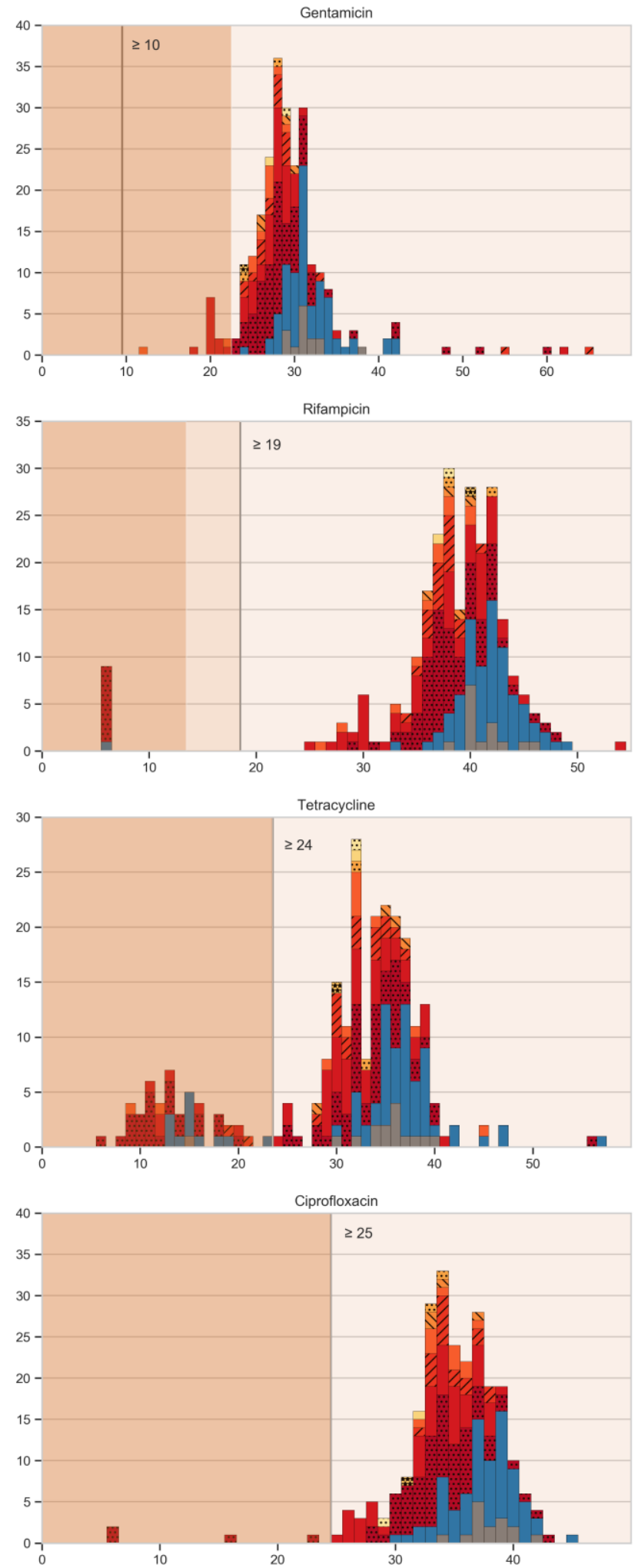

Clindamycin
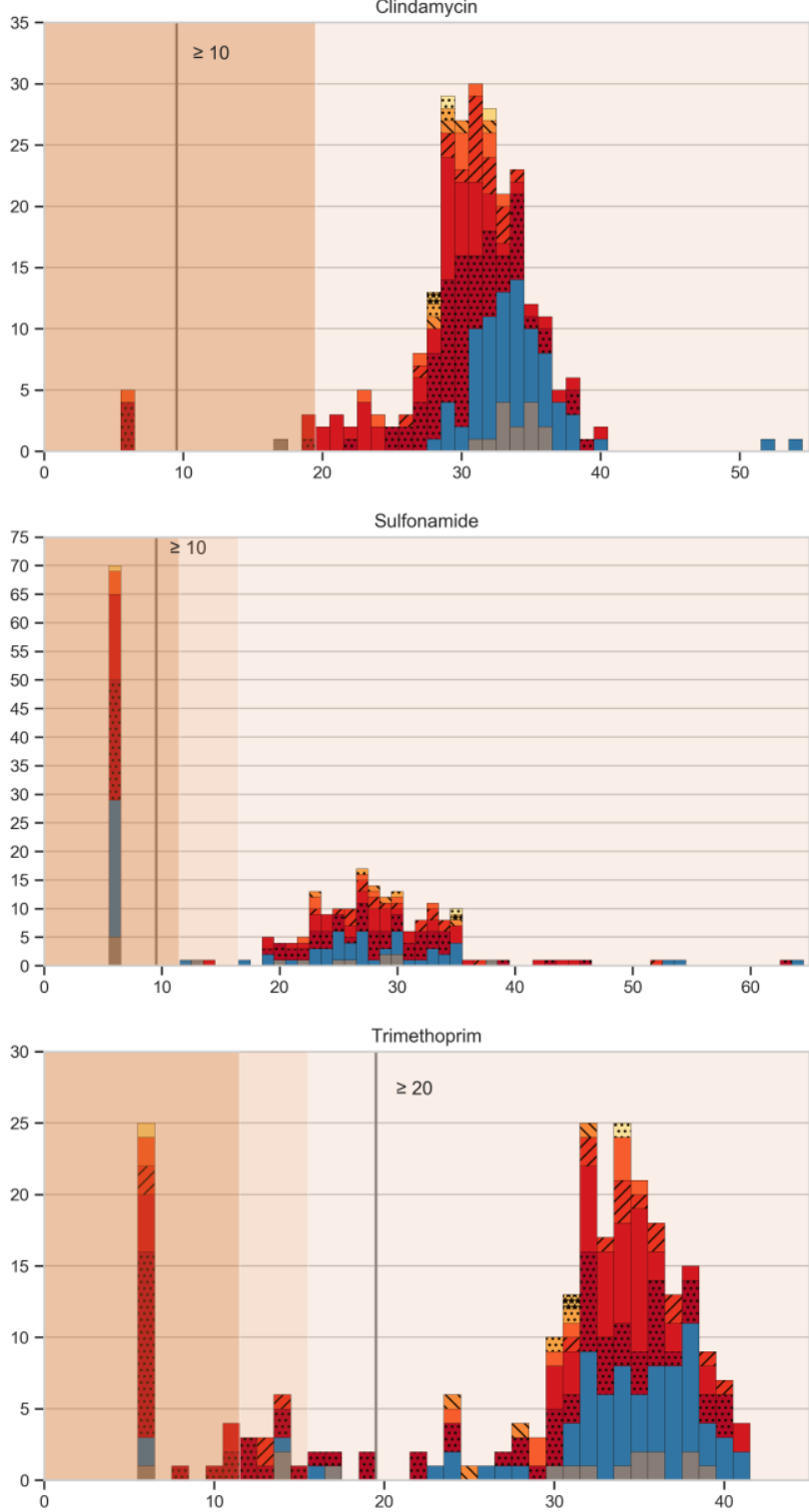

Trimethoprim-Sulfamethoxazole

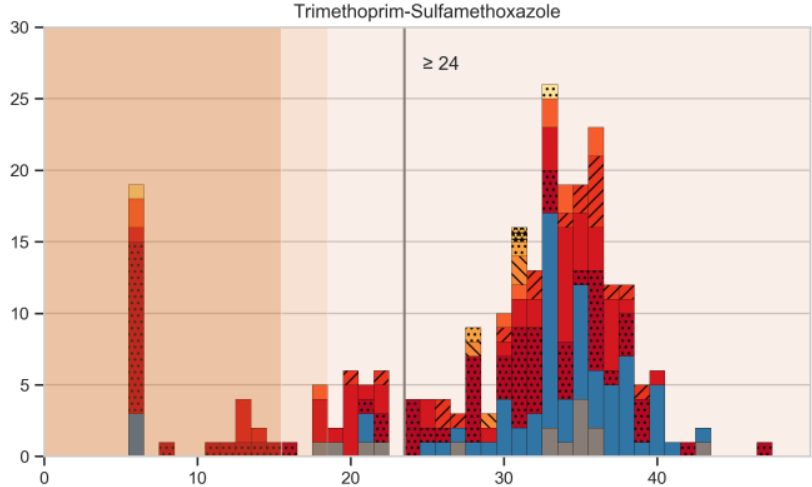




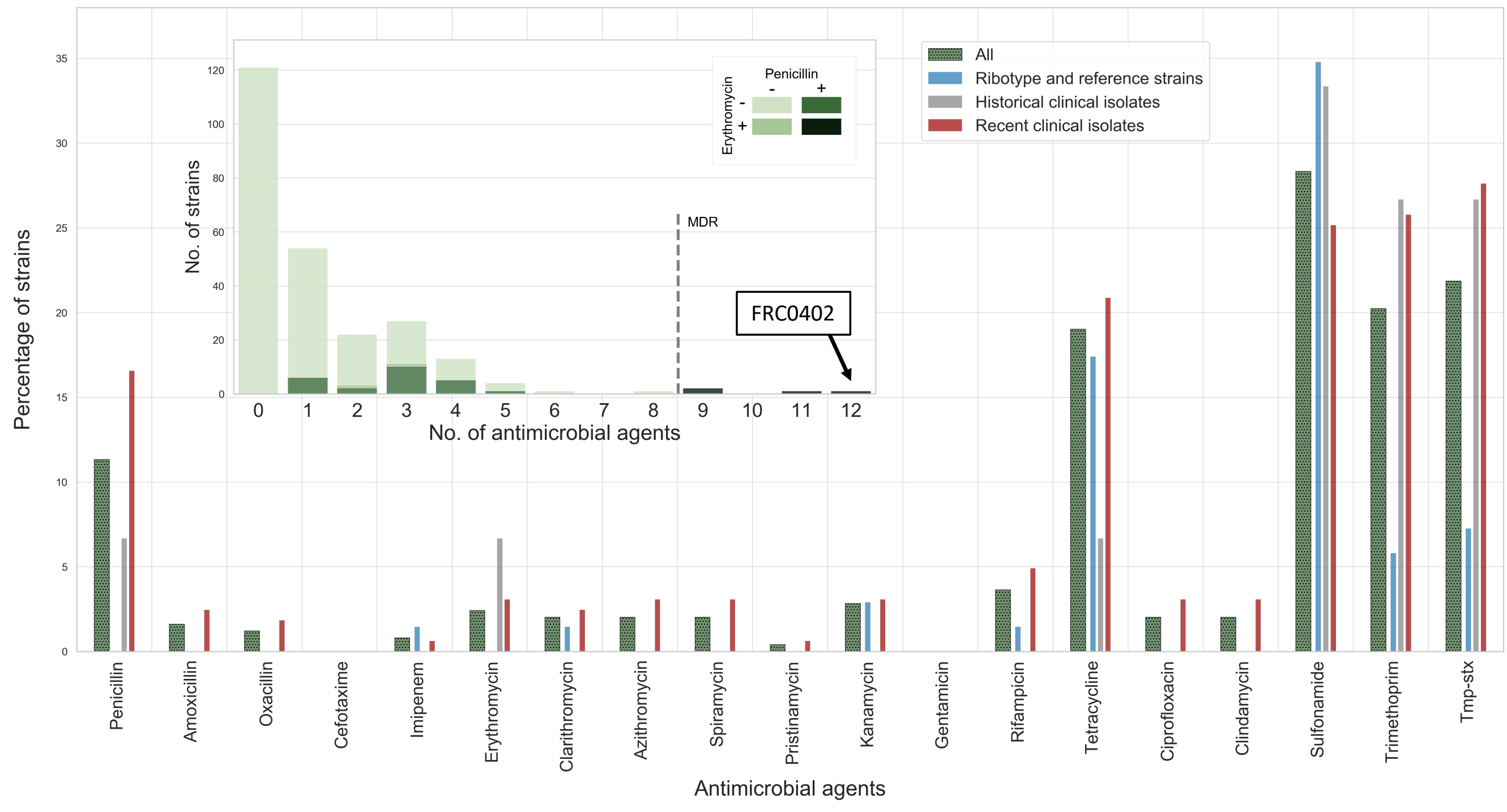


was not certified by peer review) is the author/funder, who has granted bioRxiv a license to display the preprint in perpetuity. It is made available under aCC-BY-NC-ND 4.0 International license.
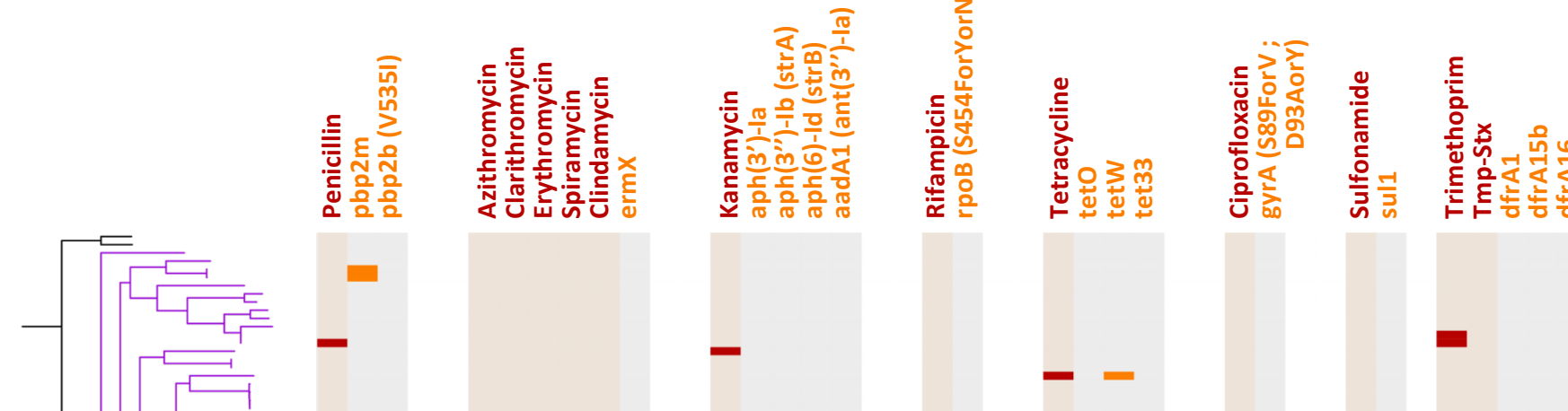
Penicillin (1 IU) Penicillin (10 IU) Amoxicillin Oxacillin Cefotaxime Imipenem Erythromycin Clarithromycin

Azithromycin Spiramycin

Pristinamycin Kanamycin Gentamicin Rifampicin Tetracycline Ciprofloxacin Clindamycin Sulfamide Trimethoprim Tmp-Stx Fosfomycin Linezolid

Vancomycine Moxifloxacin Zone diameatuâllaøleltnder aCC-BY-NC-ND 4.0 International license!
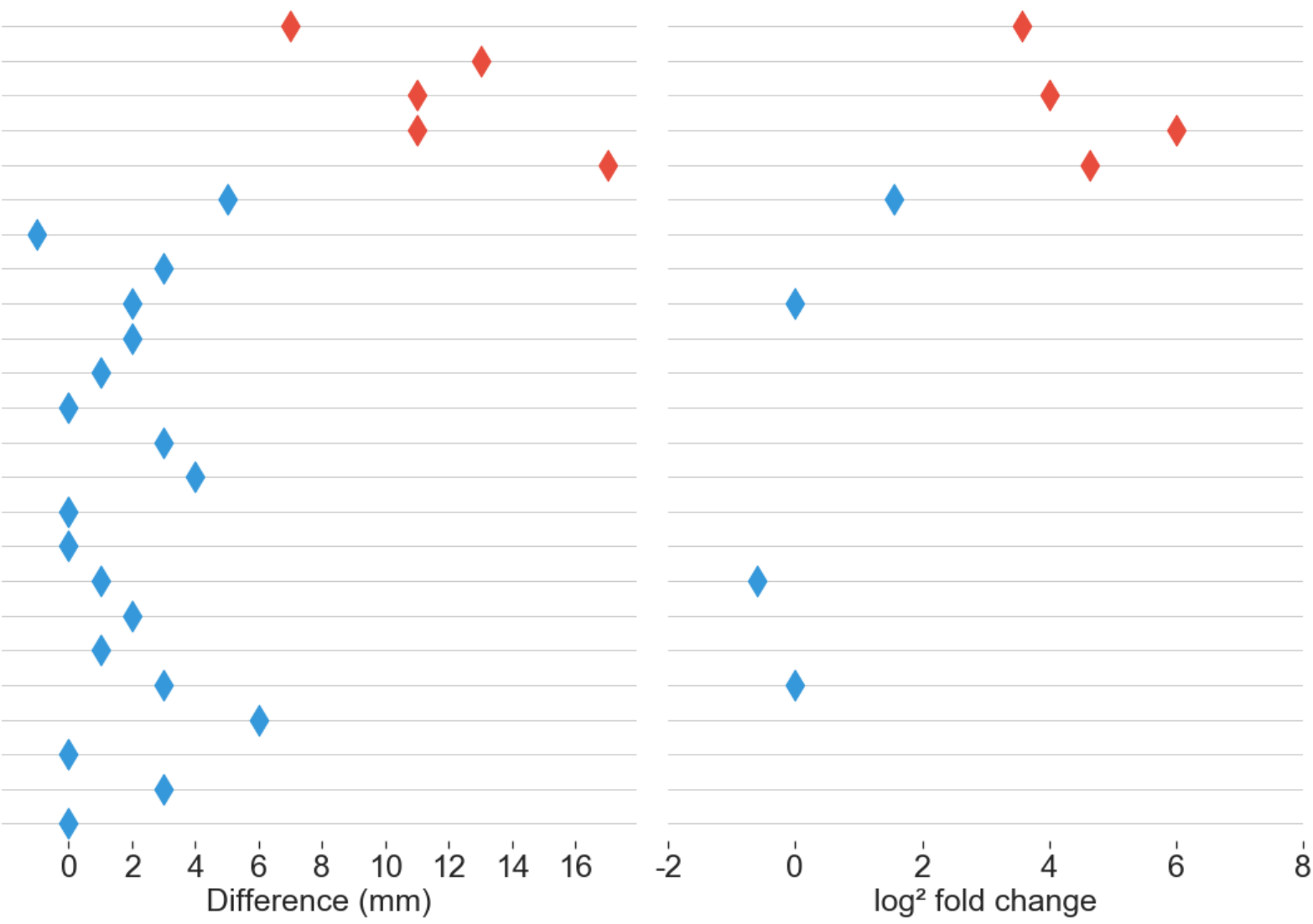


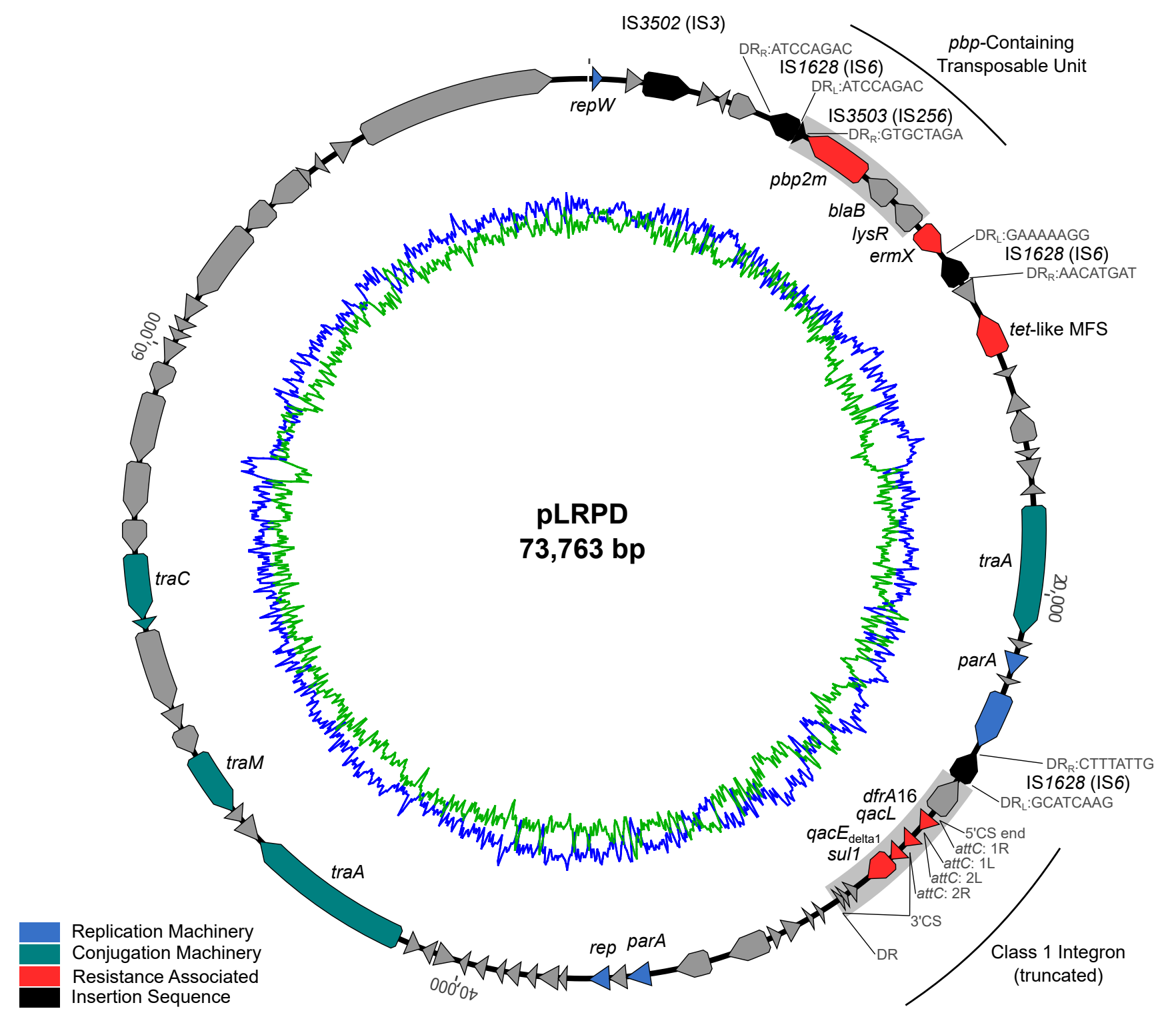

